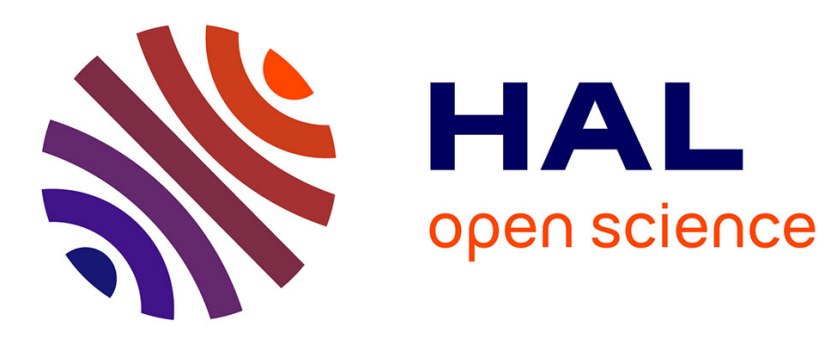

\title{
Degenerated codimension 1 crossings and resolvent estimates.
}

Thomas Duyckaerts, Clotilde Fermanian Kammerer, Thierry Jecko

\section{To cite this version:}

Thomas Duyckaerts, Clotilde Fermanian Kammerer, Thierry Jecko. Degenerated codimension 1 crossings and resolvent estimates.. Asymptotic Analysis, 2009, 65, pp.147-174. hal-00338331

\section{HAL Id: hal-00338331 \\ https://hal.science/hal-00338331}

Submitted on 12 Nov 2008

HAL is a multi-disciplinary open access archive for the deposit and dissemination of scientific research documents, whether they are published or not. The documents may come from teaching and research institutions in France or abroad, or from public or private research centers.
L'archive ouverte pluridisciplinaire HAL, est destinée au dépôt et à la diffusion de documents scientifiques de niveau recherche, publiés ou non, émanant des établissements d'enseignement et de recherche français ou étrangers, des laboratoires publics ou privés. 


\title{
Degenerated codimension 1 crossings and resolvent estimates
}

\author{
Thomas DuyckaERTS *,

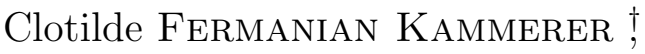 \\ Thierry JECKO ${ }^{\ddagger}$
}

\begin{abstract}
In this article, we analyze the propagation of Wigner measures of a family of solutions to a system of semi-classical pseudodifferential equations presenting eigenvalues crossings on hypersurfaces. We prove the propagation along classical trajectories under a geometric condition which is satisfied for example as soon as the Hamiltonian vector fields are transverse or tangent at finite order to the crossing set. We derive resolvent estimates for semi-classical Schrödinger operator with matrix-valued potential under a geometric condition of the same type on the crossing set and we analyze examples of degenerate situations where one can prove transfers between the modes.
\end{abstract}

\section{Introduction and main results}

\subsection{Resolvent estimate and nontrapping condition}

In this paper, we are concerned with the semi-classical Schrödinger operator acting in $L^{2}\left(\mathbb{R}^{d}, \mathbb{C}^{N}\right)$,

$$
P(\varepsilon)=-\frac{\varepsilon^{2}}{2} \Delta \cdot \operatorname{Id}+M(x)
$$

where Id is the $N \times N$ identity matrix and $M$ is a smooth, self-adjoint $N \times N$ matrix-valued potential. We require a long range behaviour of $M$ : the matrix $M$ has a limit $M_{\infty}$ at infinity and there exists $\rho>0$ such that

$$
\forall \alpha \in \mathbb{N}^{d}, \forall x \in \mathbb{R}^{d},\left|\partial_{x}^{\alpha}\left(M(x)-M_{\infty}\right)\right|=O_{\alpha}\left(\langle x\rangle^{-\rho-|\alpha|}\right),
$$

where $\langle x\rangle=\left(1+|x|^{2}\right)^{1 / 2}$. The operator $P(\varepsilon)$ is self-adjoint with domain $H^{2}\left(\mathbb{R}^{d}, \mathbb{C}^{N}\right)$ (see 43 ). We define its resolvent

$$
R(z, \varepsilon)=(P(\varepsilon)-z)^{-1}
$$

for $z$ in the resolvent set. As already seen in [32], the limiting absorption principle is valid on any interval $I \Subset\left(\left\|M_{\infty}\right\|_{\infty} ;+\infty\right)$. This means that, for any $s>1 / 2$ and any fixed $\varepsilon>0$,

$$
\sup _{\operatorname{Re} z \in I, \operatorname{Im} z \neq 0}\left\|\langle x\rangle^{-s} R(z, \varepsilon)\langle x\rangle^{-s}\right\|_{L^{2} \rightarrow L^{2}}<\infty .
$$

It turns out that the size of the previous supremum with respect to $\varepsilon$ is important for scattering theory. Roughly speaking, it is $O\left(\varepsilon^{-1}\right)$ for non resonant scattering and larger, up to some $\exp \left(c \varepsilon^{-1}\right)$

\footnotetext{
*Université de Cergy-Pontoise, UMR CNRS 8088. Partially supported by the French ANR grants OndNonLin and ControlFlux

†Université Paris Est, UMR CNRS 8050

‡Université de Cergy-Pontoise, UMR CNRS 8088. Supported by the French ANR grant BLAN08-3_ 309070
} 
with $c>0$, when a resonance is present. In the scalar case (cf. [46, 49, 50]), the non resonant situation is characterized in term of a nontrapping condition on the classical trajectories of the Hamiltonian field associated to the operator. In the matricial case, the treatment is much more complicated. Known results depend on the codimension of the eigenvalues crossings: see [29] (no crossing), 30, 32] (codimension one crossing), and [18] (codimension two crossing). Here we focus on the second situation.

For codimension one eigenvalues crossing, an assumption on the matricial structure of $M$ was used in [32]. Our aim in the present paper is to weaken this assumption as much as possible. As in [32], we focus on the situation where there exists $m \in\{1, \ldots, N\}$ such that

$$
M(x)=\sum_{j=1}^{m} E_{j}(x) \Pi_{j}(x)
$$

where, denoting by $\mathbb{C}^{N, N}$ the algebra of $N \times N$ complex matrices,

$$
E_{1}, \ldots, E_{m} \in C^{\infty}\left(\mathbb{R}^{d}, \mathbb{R}\right), \quad \Pi_{1}, \ldots, \Pi_{m} \in C^{\infty}\left(\mathbb{R}^{d}, \mathbb{C}^{N, N}\right)
$$

and for all $x$, the $\Pi_{j}(x)$ are orthogonal projectors satisfying $\Pi_{j}(x) \Pi_{k}(x)=0$ if $j \neq k$.

We will also assume that if $j \neq k, E_{j}$ differs from $E_{k}$ at least in an large open subset of $\mathbb{R}^{d}$. In 32 ] it is shown that this situation occurs under reasonable assumptions in the case of codimension 1 crossing. We will call it a smooth crossing by opposition to situations (occurring in codimension 2 or 3 crossings) where the eigenvalues and/or the eigenprojections develop singularities.

The crossing set is the closed subset of $\mathbb{R}^{d}$

$$
\mathcal{C}=\left\{x \in \mathbb{R}^{d} ; \exists j, k ; \quad E_{j}(x)=E_{k}(x) \text { and } j \neq k\right\} .
$$

We will also call crossing set the corresponding subset $\Gamma$ of $T^{*} \mathbb{R}^{d}$ :

$$
\Gamma=\left\{(x, \xi) \in T^{*} \mathbb{R}^{d}, x \in \mathcal{C}\right\}=\bigcup_{j=1}^{m} \Gamma_{j}, \text { where } \Gamma_{j}=\left\{(x, \xi) \in T^{*} \mathbb{R}^{d} ; \exists k \neq j, E_{j}(x)=E_{k}(x)\right\}
$$

We say that the crossing is of codimension 1 when $\mathcal{C}$ is a smooth codimension 1 submanifold of $\mathbb{R}^{d}$, which is equivalent to say that $\Gamma$ is a smooth codimension 1 submanifold of $T^{*} \mathbb{R}^{d}$. In the sequel we will make a weaker assumption, assuming that each of the $\Gamma_{j}$ is included in a codimension 1 submanifold $\Sigma_{j}$. This covers of course the case of codimension 1 crossing, but also the cases of codimension 2 and 3 crossing, when the crossing is smooth. We emphasize however that this smoothness condition is not satisfied for generic codimension 2 and 3 crossing, and that our result has more impact in the codimension 1 case.

Consider for $j=1 \ldots m$ the eigenvalues $\lambda_{j}(x, \xi)=\frac{1}{2}|\xi|^{2}+E_{j}(x)$ of the semi-classical symbol of $P(\varepsilon)$. We denote its Hamilton field by

$$
H_{j}(x, \xi)=\left(\nabla_{\xi} \lambda_{j}(x, \xi),-\nabla_{x} \lambda_{j}(x, \xi)\right)=\left(\xi,-\nabla_{x} E_{j}(x)\right) .
$$

Let $\Sigma_{j}\left(\infty ; H_{j}\right)$ be the set of points $(x, \xi)$ in $\Sigma_{j}$ where $H_{j}$ is tangent at infinite order to $\Sigma_{j}$. If $\Sigma_{j}$ is given (locally) by the equation $\gamma_{j}(x)=0$, then $\Sigma_{j}\left(\infty ; H_{j}\right)$ is (locally) the set of point such that $H_{j}^{k} \gamma_{j}(x)=0$, for all $k$ in $\mathbb{N}$.

Our first result gives the desired characterization of the non-resonant situation under a purely geometric condition. For the sake of clarity we do not give yet the stronger condition possible (see Theorem 1.3 below). 
Let $\rho_{j}\left(t ; x^{*}, \xi^{*}\right)=\left(x_{j}\left(t ; x^{*}, \xi^{*}\right), \xi_{j}\left(t ; x^{*}, \xi^{*}\right)\right)$ be the maximal solution of the Hamilton equation $\dot{\rho}_{j}:=d \rho_{j} / d t=H_{j}\left(\rho_{j}\right)$ with initial condition $\rho_{j}(0)=\left(x^{*} ; \xi^{*}\right)$. Observe that the flow $\rho_{j}$ is complete since $E_{j}$ is smooth. We say that $\lambda_{j}$ (or $H_{j}$ or $\rho_{j}$ ) is non-trapping at energy $E \in \mathbb{R}$ if, for all $\left(x^{*} ; \xi^{*}\right) \in \lambda_{j}^{-1}(E)$,

$$
\lim _{|t| \rightarrow \infty}\left|x_{j}\left(t ; x^{*} ; \xi^{*}\right)\right|=+\infty
$$

Theorem 1.1. Let $M$ satisfy (1.2), (1.4), (1.5), and (1.6). Assume that for all $j, \Gamma_{j}$ is included in a smooth submanifold $\Sigma_{j}$ of codimension 1 of $T^{*} \mathbb{R}^{d}$. Take an open interval $I_{0} \subset\left(\left\|M_{\infty}\right\|_{\infty} ;+\infty\right)$. Assume that, for all $j \in\{1, \cdots, m\}, \Sigma_{j}\left(\infty ; H_{j}\right) \cap \lambda_{j}^{-1}\left(I_{0}\right)$ is finite or countable. Then, the following conditions are equivalent

(i) for all $j \in\{1, \cdots, m\}$ and all $E \in I_{0}, \lambda_{j}$ is non trapping at energy $E$;

(ii) for all interval $I \Subset I_{0}$, for all $s>1 / 2$, there exist $\varepsilon_{0}>0$ and $C_{s, I}>0$ such that, for all $\epsilon \in\left(0 ; \epsilon_{0}\right]$,

$$
\sup _{\operatorname{Re} z \in I, \operatorname{Im} z \neq 0}\left\|\langle x\rangle^{-s} R(z, \varepsilon)\langle x\rangle^{-s}\right\|_{L^{2} \rightarrow L^{2}} \leq \frac{C_{s, I}}{\varepsilon} .
$$

Remark 1.2. In particular, if for all $j$ the flow $H_{j}$ is (outside $\{\xi=0\}$ ) transverse or tangent to finite order to the $j$ th crossing set $\Gamma_{j}$, then the conditions (ii) and (iii) are equivalent.

Actually, we can construct, for each $j$, some subset $\mathcal{F}_{j}$ of $\Sigma_{j}\left(\infty ; H_{j}\right)$, depending on $\Sigma_{j}$ and on $H_{j}$, such that Theorem 1.1 still holds true when the countability condition on $\Sigma_{j}\left(\infty ; H_{j}\right) \cap \lambda_{j}^{-1}\left(I_{0}\right)$ is replaced by the less restrictive condition $\mathcal{F}_{j} \cap \lambda_{j}^{-1}\left(I_{0}\right)=\emptyset$. A precise definition of the set $\mathcal{F}_{j}$ is given in Subsection 3.3 via Definitions 3.3 and 3.4 .

The conditions of Theorem 1.1 (and also of its refined version with the condition on $\mathcal{F}_{j}$ ) bear only on the eigenvalues $\lambda_{j}$ and do not depend on the projectors $\Pi_{j}$. We will now combine them with the condition on the matricial structure of $M$ which was introduced in [32]. The potential $M$ satisfies the special condition at the crossing if, for all $j \in\{1, \cdots, m\}$, the projector $\Pi_{j}$ is conormal to $\mathcal{C}$, that is, for any $x \in \mathcal{C}$ and $\xi$ in the tangent space $T_{x} \mathcal{C}$ of $\mathcal{C}$ at $x, \xi \cdot \nabla \Pi_{j}(x)=0$. Under this special condition at the crossing and some technical one at infinity (in the $x$ variable), that may be removed by the arguments of [18], it was proved in [32], that the conditions (ii) and (iii) of Theorem 1.1 are equivalent. The following theorem implies Theorem 1.1 and the result of [32.

Theorem 1.3. Let $M$ satisfy (1.2), (1.4), (1.5) and (1.6). Let $I_{0}$ be an open interval included in $\left(\left\|M_{\infty}\right\|_{\infty} ;+\infty\right)$ and assume that, for all $j \in\{1, \cdots, m\}$, the function $(x, \xi) \mapsto \xi \cdot \nabla \Pi_{j}(x)$ vanishes on $\mathcal{F}_{j} \cap \lambda_{j}^{-1}\left(I_{0}\right)$. Then, the conditions (i) and (远) of Theorem 1.1 are equivalent.

As already mentioned, for precise definition of the set $\mathcal{F}_{j}$, see Definition 3.3 and Section 3.3 . The proof of Theorem 1.3 crucially relies on the propagation result in Theorem 1.6 below.

Remark 1.4. The special condition at the crossing from 32 requires the vanishing of the functions $(x, \xi) \mapsto \xi \cdot \nabla \Pi_{j}(x)$ on points where the Hamilton field $H_{j}$ is tangent to $\mathcal{C}$. Here we assume the same vanishing on the much smaller set $\mathcal{F}_{j}$. A typical situation where $\mathcal{F}_{j}$ is not empty is when $\Sigma_{j}$ contains a piece of a trajectory of the Hamiltonian field $H_{j}$. In Section 4 , we produce an example of this kind for which Theorem 1.6 does not apply, its conclusion is even false, and the vanishing condition of Theorem 1.3 is not satisfied. This strongly suggests that Theorem 1.3 does not holds true if this vanishing condition is removed. 
Remark 1.5. Thanks to (1.2), there exists $\lambda_{0}>0$ such that the function $(x, \xi) \mapsto x \cdot \xi \cdot$ Id is a global escape function at all energy $\lambda \in\left(\lambda_{0},+\infty\right)$ for the matrix-valued symbol $p:(x, \xi) \mapsto|\xi|^{2} \cdot \operatorname{Id}+M(x)$ in the sense of [30]. By Theorem 2.3 in [30] (which actually holds true with the same proof for all matricial dimension $N$ ), we get (ii) of Theorem 1.1 for $I_{0}=\left(\lambda_{0} ;+\infty\right)$. Assume now that the assumptions of Theorem 1.3 are satisfied. We derive from Theorem 1.3 that all energy $\lambda \in\left(\lambda_{0},+\infty\right)$ is non trapping for all fields $H_{j}$. Then, as in [18], one can upperbound the resolvent in (1.8) by $C \varepsilon^{-1} \lambda^{-1 / 2}$, where $C$ only depends on $\lambda_{0}$. Arguing as in [18], one gets local in time $H^{s}$ estimates, smoothing effect and Strichartz estimates, and one can prove existence and uniqueness of solutions of non-linear semi-classical Schrödinger equation with matrix-valued potentials in a situation where the potential do not decrease at infinity.

\subsection{Codimension 1 crossings and Wigner measures}

A key argument in the proof of Theorem 1.3 is a result on propagation of Wigner measures in presence of degenerated codimension 1 crossing. We next present this result, which is of interest by itself. We will work in a general pseudodifferential framework, as in [9, [10], [17 and [14]. This framework contains the one of Theorem 1.3. We refer to [9, Section 2] for other applications.

We first recall a few facts about Wigner measures (see [23], [24], [25], [37] or the survey [6]). Consider $\left(\psi^{\varepsilon}\right)_{\varepsilon>0}$ a bounded family in the weighted $L^{2}$-space $L_{-s}^{2}\left(\mathbb{R}^{d} ; \mathbb{C}^{N}\right):=L^{2}\left(\mathbb{R}^{d} ; \mathbb{C}^{N} ;\langle x\rangle^{s} d x\right)$, for some $s \geq 0$. Then there exists a positive hermitian Radon measure $\mu$ and a sequence $\varepsilon_{k}$ going to 0 as $k$ goes to $+\infty$ such that

$$
\forall a \in \mathcal{C}_{0}^{\infty}\left(\mathbb{R}^{2 d} ; \mathbb{C}^{N, N}\right), \quad\left(\mathrm{op}_{\varepsilon_{k}}(a) \psi^{\varepsilon_{k}}, \psi^{\varepsilon_{k}}\right) \underset{k \rightarrow+\infty}{\longrightarrow} \operatorname{tr} \int_{\mathbb{R}^{2 d}} a(x, \xi) \mathrm{d} \mu(x, \xi) .
$$

Here $\operatorname{op}_{\varepsilon}(a)$ denotes the semi-classical Weyl quantization of $a$, namely the operator defined by

$$
\mathrm{op}_{\varepsilon}(a) f(x)=\int_{\mathbb{R}^{2 d}} a\left(\frac{x+x^{\prime}}{2}, \xi\right) \mathrm{e}^{\frac{i}{\varepsilon} \xi \cdot\left(x-x^{\prime}\right)} f\left(x^{\prime}\right) \frac{\mathrm{d} x^{\prime} \mathrm{d} \xi}{(2 \pi \varepsilon)^{d}} .
$$

We recall here that, when $a \in \mathcal{C}_{0}^{\infty}\left(\mathbb{R}^{2 d}\right)$, 1.10$)$ defines an operator which is continuous, uniformly with respect to $\varepsilon$, from $L_{-s}^{2}$ to $L^{2}$ and from $L_{\text {loc }}^{2}$ to $L_{\text {loc }}^{2}$. At many places in this paper, we shall use well-known properties of semi-classical pseudodifferential calculus. See [12, 38] for details.

The matrix-valued measure $\mu$ describes the oscillation of the sequence $\left(\psi^{\varepsilon_{k}}\right)_{k}$ which are exactly of size $1 / \varepsilon_{k}$ or smaller. Such measure is called a Wigner measure associated to the family $\left(\psi^{\varepsilon}\right)_{\varepsilon}$. It is a positive hermitian matrix-valued measure in the sense that for all scalar positive smooth compactly supported test-function $a$, the quantity $\int a(x, \xi) \mathrm{d} \mu(x, \xi)$ is a positive hermitian matrix.

Let $m \in\{1, \ldots N\}$ and consider $m$ real-valued smooth functions $\left(\lambda_{j}\right)_{j=1 \ldots m}, m$ matrix valued smooth functions $\left(\Pi_{j}\right)_{j=1 \ldots m}$ on $\mathbb{R}^{2 d}$. Again we assume that the $\Pi_{j}(x, \xi)$ are orthogonal projectors satisfying $\Pi_{j} \Pi_{k} \equiv 0$ if $j \neq k$. Let

$$
Q(x, \xi)=\sum_{j=1}^{m} \lambda_{j}(x, \xi) \Pi_{j}(x, \xi) .
$$

We will also assume that $Q$ satisfies, for some real $r_{1}$ and $r_{2}$,

$$
\forall \alpha, \beta \in \mathbb{N}^{d}, \exists C_{\alpha, \beta}>0 ;\left|\partial_{x}^{\alpha} \partial_{\xi}^{\beta} Q(x, \xi)\right| \leq C_{\alpha, \beta}(1+|x|)^{r_{1}-|\alpha|}(1+|\xi|)^{r_{2}-|\beta|} .
$$

Let us mention that the result and the computations of this subsection are essentially local, so one can probably relax assumption (1.11) on $Q$. 
We consider a family $\left(\psi^{\varepsilon}\right)_{\varepsilon>0}$ such that for all $\varepsilon, \psi^{\varepsilon}$ belongs to the domain of op ${ }_{\varepsilon}(Q)$. We assume that the family is bounded in $L^{2}\left(\mathbb{R}^{d} ; \mathbb{C}^{N}\right)$ and satisfies, on some open subset $\Omega$ of $\mathbb{R}^{d}$,

$$
\mathrm{op}_{\varepsilon}(Q) \psi^{\varepsilon}=o(\varepsilon) \text { in } L^{2}\left(\Omega, \mathbb{C}^{N}\right), \text { as } \varepsilon \rightarrow 0 .
$$

We define again the crossing set $\Gamma$ by

$$
\Gamma=\bigcup_{j=1}^{m} \Gamma_{j}, \quad \Gamma_{j}=\left\{(x, \xi) \in T^{*} \Omega ; \exists k \neq j, \lambda_{j}(x, \xi)=\lambda_{k}(x, \xi)\right\}
$$

and we assume that for all $j, \Gamma_{j}$ is included in a codimension 1 submanifold $\Sigma_{j}$.

As above, for $1 \leq j \leq m$, we denote by $H_{j}$ the Hamiltonian vector fields associated with the functions $\lambda_{j}$ and by $\Sigma_{j}\left(\infty ; H_{j}\right)$ the set of points $(x, \xi)$ in $\Sigma_{j}$ where $H_{j}$ is tangent at infinite order to $\Sigma_{j}$. We can define the previous closed subset $\mathcal{F}_{j}$ of $\Sigma_{j}\left(\infty ; H_{j}\right)$, that contains all the characteristic curves of $H_{j}$ that are included in $\Sigma_{j}$. Recall that $\mathcal{F}_{j}$ is empty if $\Sigma_{j}\left(\infty ; H_{j}\right)$ is at most countable. Here again, we refer to Definitions 3.3 and 3.4 for a precise definition.

Finally, for smooth matrix-valued functions $a, b$ on $T^{*} \mathbb{R}^{d}$, the Poisson bracket $\{a, b\}$ is the matrixvalued function defined by $\nabla_{\xi} a \cdot \nabla_{x} b-\nabla_{x} a \cdot \nabla_{\xi} b$. Setting, for all $j$,

$$
\begin{aligned}
& B_{j}=-\frac{1}{2}\left\{\Pi_{j}, Q+\lambda_{j} \mathrm{Id}\right\} \\
& R_{j}=\left[\left\{\lambda_{j}, \Pi_{j}\right\}, \Pi_{j}\right]+\frac{1}{2} \sum_{k}\left(\lambda_{k}-\lambda_{j}\right) \Pi_{j}\left\{\Pi_{k}, \Pi_{k}\right\} \Pi_{j},
\end{aligned}
$$

our propagation result is the following

Theorem 1.6. Let $\mu$ be any Wigner measure of the family $\left(\psi^{\varepsilon}\right)_{\varepsilon>0}$ and $j \in\{1, \cdots, m\}$. Let $\omega$ be a bounded open neighborhood of some $\left(x^{*}, \xi^{*}\right) \in \Omega \times \mathbb{R}^{d}$ such that $\bar{\omega} \subset \Omega \times \mathbb{R}^{d}$. Assume that $B_{j}$ vanishes on $\mathcal{F}_{j} \cap \omega$. Then as distributions on $\omega$,

$$
H_{j}\left(\Pi_{j} \mu \Pi_{j}\right)=\left[R_{j}, \Pi_{j} \mu \Pi_{j}\right] .
$$

In particular, the trace of $\Pi_{j} \mu \Pi_{j}$ is invariant under the flow of $H_{j}$.

Remark 1.7. If $\left(x^{*}, \xi^{*}\right) \notin \Sigma_{j}$, then (1.16) holds true on small enough $\omega$ without any assumption on $B_{j}$.

Remark 1.8. Replacing the operator $\mathrm{op}_{\varepsilon}(Q)$ by $D_{t}-\mathrm{op}_{\varepsilon}(Q)$, one can show that any Wigner measure $\mu$ of the time-dependent family $\left(\psi^{\varepsilon}\right)_{\varepsilon>0}$ satisfies

$$
\left(\partial_{t}-H_{j}\right)\left(\Pi_{j} \mu \Pi_{j}\right)=\left[R_{j}, \Pi_{j} \mu \Pi_{j}\right] .
$$

Remark 1.9. Theorem 1.6 actually implies the same theorem for $\left(\psi^{\varepsilon}\right)_{\varepsilon>0}$ bounded in some $L_{-s}^{2}$ with $s>0$ and satisfying (1.12) with $L^{2}\left(\Omega, \mathbb{C}^{N}\right)$ replaced by $L_{l o c}^{2}\left(\Omega, \mathbb{C}^{N}\right)$ (see Remark 3.1).

Remark 1.10. If $Q(x, \xi)=\frac{1}{2}|\xi|^{2}+M(x)$ with $M$ as in (1.4), then $\mathrm{op}_{\varepsilon}(Q)=P(\varepsilon)$ and

$$
B_{j}(x, \xi)=\frac{1}{2} \xi \cdot \nabla \Pi_{j}(x), \quad R_{j}=\left[\xi \cdot \nabla \Pi_{j}(x), \Pi_{j}(x)\right] .
$$

Motivated by the comments in Remark 1.4, we also analyse in Section 1 a strongly degenerated situation which is excluded in Theorem 1.6. Finally, in the Appendix, we give a microlocal normal form which should be of interest for studying at any order in $\varepsilon$ a solution to a partial differential equation close to a non-degenerated point in a codimension 1 crossing. 


\subsection{Comments on the results}

The analysis of the propagation of Wigner measures in presence of eigenvalue crossing has been the subject of intensive works in the last ten years. The existing results are usually devoted to generic situations where the Hamiltonian vector fields associated with the eigenvalues are transverse to the crossing set (see 14] 17]). Theorem 1.6 covers more general situations where the Hamiltonian fields may be tangent to this set. To our knowledge, it is the first result on the propagation of Wigner measure in presence of eigenvalue crossing in a degenerated situation. We point out that G. Hagedorn gave an important pioneer contribution to such propagation phenomena in [27], where he presented a systematic study of the propagation of a Gaussian wave packet through generic crossings of various codimension. We also want to quote the thesis of U. Karlsson 33] for the construction of a parametrix in the presence of a smooth eigenvalue crossing and the work of M. Brassart [2] who studied codimension 1 eigenvalue crossings in a periodic situation.

Finally, let us mention that, for codimension 2 and 3 crossings, normal forms have been obtained by Y. Colin de Verdière in [9] and [10]. These important results yield a very detailed description of the solution close to some generic point in the crossing. We give here a similar normal form for codimension 1 crossing in the Appendix under a non-degeneracy condition consisting in assuming the transversality of the classical trajectories to the crossing set and the fact that the gap between the eigenvalues vanish at order 1 on the crossing set.

Concerning the resolvent estimates, there are many results for smooth, scalar Schrödinger operators (see [3, 13, 21, 22, 31, 46, 47, 50]). For less regular but still scalar potential, we quote 77, 8]. In the matricial case, there are rather few results since propagation results like Theorem 1.6 are difficult to obtain. Indeed, it is rather involved to control the influence of eigenvalues crossings. We quote [18, 29, 30, 32. On the related question of existence of resonances for matrix Schrödinger operators, we mention [20, 42, where only $2 \times 2$ matrix operators are considered. Notice that the mentioned resolvent estimates are of great interest for semiclassical, molecular scattering theory (a theory for chemical reactions), since a matricial Schrödinger operator is a toy model for real molecules.

Let us say a few words about the proofs. The proof of Theorem 1.3 follows the strategy of [32]. The necessity of the non trapping condition is proved following the method of X.P. Wang in 50 , as adapted in [18] using Wigner measures (in particular without the condition at infinity of [32]). We refer to [1] for a similar proof. The sufficiency of the non trapping condition is, as in [31, 32], obtained by contradiction using the method of N. Burq in [3], which is inspired by an argument of G. Lebeau in [36], and also by the use of a rescaled Mourre estimate at space infinity derived in [32] (see also 31]). The idea is to use Wigner measure to show that some particular sequence, which negates the resolvent bound, tends to 0 in $L_{\text {loc }}^{2}$. One step of the proof is to show, using the long-range condition (1.2), that the Wigner measure is compactly supported. This is done in 32] and follows from the rescaled Mourre estimate. The new ingredient in the proof of Theorem 1.3 is the propagation of the Wigner measures at the crossing set, which follows from Theorem 1.6, under much weaker assumptions than in [32]. The proof of this result relies on an induction on the order of tangency of the flow, reminiscent of an argument due to R.B. Melrose and J. Sjöstrand in the context of propagation of singularities of boundary value problems [39]. A standard induction, as in [39], gives the propagation around points of the crossing set where the flow has a finite order contact with the crossing set. Here we use a transfinite induction to get the propagation in a larger set that may also contains some points with infinite order contact. Finally, the study of the degenerated situation in Section 1 relies on the use of a two-scale Wigner measure (see [40, 16]) to describe more precisely the behaviour of the Wigner measure at the crossing set. 
The organization of the paper is the following. The two steps of the proof of Theorem 1.3, which are by now classical, are quickly sketched in Section 2, assuming that Theorem 1.6 is true. The latter is proved in Section 3. Then, in Section 4, we analyze the strongly degenerated situation mentioned above. Finally, in the Appendix, a normal form is given in a non-degenerate situation.

Acknowledgment. The second author wish to thank Patrick Gérard for fruitful discussions on the subject. The first author wish to thank Thomas Chomette and Gilles Godefroy for clarifications on ordinal numbers.

\section{Non-trapping condition and resolvent estimate}

Here we assume Theorem 1.6 true and we prove Theorem 1.3. We work under the assumptions of Theorem 1.3. We do not need to understand what is the set $\mathcal{F}_{j}$. Its meaning is relevant for the proof of Theorem 1.6 only.

\subsection{The necessity of the non-trapping condition}

Let us first focus on the necessity of the non-trapping condition. We adapt the arguments in [18], which are inspired by [50]. Let $E \in I_{0}$ and $\theta \in \mathcal{C}_{0}^{\infty}\left(I_{0}\right)$ with $\theta=1$ near $E$. Let $I$ be the support of $\theta$. The resolvent estimate (1.8) implies that, for $s>1 / 2$ and uniformly w.r.t. $\varepsilon \in] 0, \varepsilon_{0}[$, the function $\langle x\rangle^{-s}$ is $P(\varepsilon)$-smooth on $I$ (see Theorem XIII 25 in 44 ). Therefore there exists a constant $C_{0}$ such that for any $\varepsilon \in] 0, \varepsilon_{0}\left[\right.$ and any $\psi \in L^{2}$

$$
\int_{\mathbb{R}}\left\|\langle x\rangle^{-s} \theta(P(\varepsilon)) \mathrm{e}^{-i \frac{t}{\varepsilon} P(\varepsilon)} \psi\right\|_{L^{2}}^{2} \mathrm{~d} t \leq C_{0}\|\psi\|_{L^{2}}^{2}
$$

We are going to prove that if $\left(x_{j}(t), \xi_{j}(t)\right)_{t \in \mathbb{R}}$ is a classical trajectory of $H_{j}$ of energy $E$, that is contained in $\lambda_{j}^{-1}(E)$, then

$$
\forall T>0, \quad \int_{-T}^{+T}\left\langle x_{j}(t)\right\rangle^{-s} \mathrm{~d} t \leq C_{0} .
$$

This property implies that $E$ is non-trapping (cf. [50]). Let us prove (2.2). Consider a trajectory $\left(x_{j}(t), \xi_{j}(t)\right)_{t \in \mathbb{R}}$ of $H_{j}$ of energy $E$ and let $\left(\psi_{0}^{\varepsilon}\right)_{\varepsilon>0}$ be a by one bounded family in $L^{2}\left(\mathbb{R}^{2}, \mathbb{C}^{N}\right)$ having only one Wigner measure $\mu_{0}$ such that

$$
\mu_{0}=c_{0} \delta\left(x-x_{j}(0)\right) \otimes \delta\left(\xi-\xi_{j}(0)\right) \Pi_{j}(x) .
$$

One can actually choose coherent states microlocalized at $\left(x_{j}(0), \xi_{j}(0)\right)$, for instance. We consider $\psi^{\varepsilon}(t)=\mathrm{e}^{-i \frac{t}{\varepsilon} P(\varepsilon)} \psi_{0}^{\varepsilon}$. The family $\left(\psi^{\varepsilon}\right)_{\varepsilon>0}$ satisfies

$$
\frac{\varepsilon}{i} \partial_{t} \psi^{\varepsilon}+P(\varepsilon) \psi^{\varepsilon}=0, \quad \psi_{\mid t=0}^{\varepsilon}=\psi_{0}^{\varepsilon} .
$$

Let $\mu_{t}$ be a Wigner measure of $\left(\psi^{\varepsilon}(t)\right)_{\varepsilon>0}$. Let $k \neq j$. By Theorem 1.6, the measure $\Pi_{k} \mu_{t} \Pi_{k}$ satisfies a linear differential equation with initial data $\Pi_{k} \mu_{0} \Pi_{k}=0$, thus it is zero. Since $\Pi_{k} \mu_{t} \Pi_{j}$ is absolutely continuous w.r.t. $\Pi_{k} \mu_{t} \Pi_{k}$ and $\Pi_{j} \mu_{t} \Pi_{j}$, it is also zero. Thus $\mu_{t}=\Pi_{j} \mu_{t} \Pi_{j}$. Theorem 1.6 yields

$$
\operatorname{tr} \mu(t, x, \tau, \xi)=c_{0} \delta\left(x-x_{j}(t)\right) \otimes \delta\left(\xi-\xi_{j}(t)\right) \otimes \delta\left(\tau+\lambda_{j}(x, \xi)\right) \otimes d t
$$


Let $p(x, \xi)=\frac{|\xi|^{2}}{2}+M(x)$ be the symbol of $P(\varepsilon)$. Take $T>0$ and a non-negative, smooth, compactly supported, scalar function $(t, x, \xi) \mapsto a(t, x, \xi)$ such that

$$
\forall t \in[-T, T], a\left(t, x_{j}(t), \xi_{j}(t)\right)=1 \text { and }(1-\theta(p)) a(t, \cdot, \cdot)=0 .
$$

By (2.1) and the fact that $\left\|\psi_{0}^{\varepsilon}\right\|_{L^{2}} \leq 1$, for all $\varepsilon$,

$$
\int_{-T}^{+T}\left(\operatorname{op}_{\varepsilon}(a(t, x, \xi))\langle x\rangle^{-s} \theta(P(\varepsilon)) \psi^{\varepsilon}(t) \mid\langle x\rangle^{-s} \theta(P(\varepsilon)) \psi^{\varepsilon}(t)\right)_{L^{2}} \mathrm{~d} t \leq C_{0} .
$$

We first observe that, uniformly w.r.t. $t \in[-T ; T]$,

$$
\langle x\rangle^{-s} \mathrm{op}_{\varepsilon}(a(t, x, \xi))\langle x\rangle^{-s}=\mathrm{op}_{\varepsilon}\left(\langle x\rangle^{-2 s} a(t, x, \xi)\right)+o(1) \text { in } \mathcal{L}\left(L^{2}\left(\mathbb{R}^{d}\right)\right) .
$$

Then, the functional calculus and (2.4) give, in $L^{2}\left(\mathbb{R}^{d}\right)$,

$$
\mathrm{op}_{\varepsilon}\left(\langle x\rangle^{-2 s} a(t, x, \xi)\right)(1-\theta(P(\varepsilon)))=\mathrm{op}_{\varepsilon}\left(\langle x\rangle^{-2 s} a(t, x, \xi)(1-\theta(p(x, \xi)))\right)+o(1)=o(1)
$$

where by matricial functional calculus for fixed $(x, \xi), \theta(p(x, \xi))=\sum_{1 \leq j \leq m} \theta\left(\lambda_{j}(x, \xi)\right) \Pi_{j}(x)$. Therefore, writing $\psi^{\varepsilon}(t)=\theta(P(\varepsilon)) \psi^{\varepsilon}(t)+(\operatorname{Id}-\theta(P(\varepsilon))) \psi^{\varepsilon}(t)$,

$$
\begin{aligned}
\int_{-T}^{+T}\left(\mathrm{op}_{\varepsilon}(a(t, x, \xi))\langle x\rangle^{-s} \psi^{\varepsilon}(t) \mid\langle x\rangle^{-s} \psi^{\varepsilon}(t)\right)_{L^{2}} \mathrm{~d} t \\
=\int_{-T}^{+T}\left(\mathrm{op}_{\varepsilon}(a(t, x, \xi))\langle x\rangle^{-s} \theta(P(\varepsilon)) \psi^{\varepsilon}(t) \mid\langle x\rangle^{-s} \theta(P(\varepsilon)) \psi^{\varepsilon}(t)\right)_{L^{2}} \mathrm{~d} t+o(1) .
\end{aligned}
$$

Using (2.5) and passing to the limit $\varepsilon \rightarrow 0$, we get, since $a$ is scalar and satisfies (2.4),

$$
\begin{aligned}
\int_{-T}^{+T} \int a(t, x, \xi)\langle x\rangle^{-2 s} \operatorname{tr} \mathrm{d} \mu_{t}(x, \xi) \mathrm{d} t & =\int_{-T}^{+T} a\left(t, x_{j}(t), \xi_{j}(t)\right)\left\langle x_{j}(t)\right\rangle^{-2 s} \mathrm{~d} t \\
& =\int_{-T}^{+T}\left\langle x_{j}(t)\right\rangle^{-2 s} \mathrm{~d} t \leq C_{0},
\end{aligned}
$$

whence (2.2).

\subsection{The sufficiency of the non-trapping condition}

Now we assume that the non trapping condition is fulfilled on some open interval $I_{0}$ included in $\left(\left\|M_{\infty}\right\|_{\infty} ;+\infty\right)$ and we prove the resolvent estimate (1.8) by contradiction. Suppose that, for some interval $I \Subset I_{0}$, some $s>1 / 2$, and some $\epsilon_{0}>0$, (1.8) is false. Then it is shown in 32 that the following situation occurs: there exist a sequence $\left(\epsilon_{n}\right) \in\left(0 ; \epsilon_{0}\right)^{\mathbb{N}}$ tending to 0 , a sequence $\left(f_{n}\right)$ of $H^{2}\left(\mathbb{R}^{d}\right)$-functions, a sequence $\left(z_{n}\right) \in \mathbb{C}^{\mathbb{N}}$ such that $\mathcal{R} e z_{n} \rightarrow E \in I,\left(\mathcal{I} m z_{n}\right) / \epsilon_{n} \rightarrow 0,\left(f_{n}\right)$ is bounded in $L_{-s}^{2}\left(\mathbb{R}^{d}\right)$, has a unique Wigner measure $\mu$, and the $L^{2}$-norm $\left\|\langle x\rangle^{s}\left(P\left(\varepsilon_{n}\right)-z_{n}\right) f_{n}\right\|_{L^{2}}$ is a $o\left(\varepsilon_{n}\right)$. Furthermore, the long range condition (1.2) implies the existence of some $R>0$ such that

$$
\lim _{n \rightarrow \infty} \int_{|x| \geq R}\langle x\rangle^{-2 s}\left|f_{n}(x)\right|^{2} d x=0 .
$$

This implies in particular that $\mu$ is nonzero and supported in the compact set

$$
\left(\bigcup_{1 \leq j \leq m} \lambda_{j}^{-1}(E)\right) \cap\{(x, \xi) ;|x| \leq R\} .
$$


Taking $\tau \in \mathcal{C}_{0}^{\infty}\left(\mathbb{R}^{d}\right)$ such that $\tau=1$ near the set $\left\{x \in \mathbb{R}^{d} ;|x| \leq R\right\}$, one can show, by direct computations, that the sequence $\left(g_{n}\right)$, defined by $g_{n}=\tau f_{n}$, is bounded in $L^{2}\left(\mathbb{R}^{d}\right)$, has $\mu$ as unique Wigner measure, and satisfies $\left\|\left(P\left(\varepsilon_{n}\right)-\mathcal{R} e z_{n}\right) g_{n}\right\|=o\left(\varepsilon_{n}\right)$ (as in [7]). In view of Remark 1.10, we can apply Theorem 1.6 to $\left(g_{n}\right)$. Since, for all $j$, the scalar measure $\operatorname{tr} \prod_{j} \mu \Pi_{j}$ is compactly supported and invariant under the flow of $H_{j}$, the non-trapping condition for $H_{j}$ imposes that $\operatorname{tr} \Pi_{j} \mu \Pi_{j}=0$. Since the diagonal terms of the matricial measure $\Pi_{j} \mu \Pi_{j}$ (recall that $\Pi_{j}$ may have rank $>1$ ) are non negative, they all vanish. Since the off-diagonal terms of $\Pi_{j} \mu \Pi_{j}$ are absolutely continuous w.r.t. the diagonal terms, they also vanish. Thus, $\Pi_{j} \mu \Pi_{j}=0$ for all $j$. Since the measures $\Pi_{k} \mu \Pi_{j}$ with $k \neq j$ are absolutely continuous w.r.t. $\Pi_{k} \mu \Pi_{k}$ and $\Pi_{j} \mu \Pi_{j}$, they all vanish and $\mu=0$, yielding the desired contradiction.

Remark 2.1. Under the previously mentioned special condition at the crossing, it is shown in [32] that, for all $j$, the matricial measure $\Pi_{j} \mu \Pi_{j}$ is actually invariant under the flow of $H_{j}$. Here we use a weaker information namely the propagation result in Theorem 1.6.

The derivation of the properties of $\left(g_{n}\right)$ is quite immediate here. In [7] it is more complicated due to the presence of singularities.

\section{Propagation of Wigner measures}

In this section, we prove Theorem 1.6. First we explain in the next remark why the extension of Theorem 1.6 announced in Remark 1.9 holds true.

Remark 3.1. Assume that $\left(\psi^{\varepsilon}\right)_{\varepsilon>0}$ bounded in some $L_{-s}^{2}$ with $s>0$ and satisfy (1.12) with $L^{2}\left(\Omega, \mathbb{C}^{N}\right)$ replaced by $L_{\text {loc }}^{2}\left(\Omega, \mathbb{C}^{N}\right)$. Given a bounded open subset $\omega$ of $\Omega$ with $\bar{\omega} \subset \Omega$, take functions $\tau_{1}, \tau_{2} \in \mathcal{C}_{0}^{\infty}(\Omega)$ such that $\tau_{1} \tau_{2}=\tau_{2}$ and $\tau_{2}=1$ near $\omega$. Let $Q_{1}$ be defined by $\tau_{2} o p_{\varepsilon}(Q)=o p_{\varepsilon}\left(Q_{1}\right)$ and $\phi^{\varepsilon}=\tau_{1} \psi^{\varepsilon}$. Then we can apply Theorem 1.6 for $\left(\phi^{\varepsilon}\right)_{\varepsilon>0}$ and $Q_{1}$. This yields (1.16) for any Wigner measure of $\left(\psi^{\varepsilon}\right)_{\varepsilon>0}$ on this $\omega$.

Without loss of generality, we may assume that $\left(\psi^{\varepsilon}\right)_{\varepsilon}$ has only one Wigner measure $\mu$. It is a straightforward consequence of the functional calculus and equation (1.12) that

$$
\operatorname{supp} \mu \subset\{\operatorname{det}(Q(x, \xi))=0\}=\bigcup_{1 \leq j \leq N}\left\{\lambda_{j}(x, \xi)=0\right\} .
$$

Let us consider $\chi \in \mathcal{C}_{0}^{\infty}\left(\Omega \times \mathbb{R}^{d} ; \mathbb{R}\right)$ such that $\chi=1$ on $\bar{\omega}$. We set $\psi_{j}^{\varepsilon}:=\mathrm{op}_{\varepsilon}\left(\chi \Pi_{j}\right) \psi^{\varepsilon}$. Notice that $\Pi_{j}$ may behave badly at infinity and may not belong to a good symbolic class. However $\chi \Pi_{j} \in \mathcal{C}_{0}^{\infty}\left(\mathbb{R}^{d}\right)$ and the family $\left(\psi_{j}^{\varepsilon}\right)_{\varepsilon>0}$ is bounded in $L^{2}$. The Wigner measure of $\left(\psi_{j}^{\varepsilon}\right)_{\varepsilon}$ is $\mu_{j}=\chi^{2} \Pi_{j} \mu \Pi_{j}$. Denoting by $\mu_{i j}$ the joint measure of $\left(\psi_{j}^{\varepsilon}\right)_{\varepsilon}$ and $\left(\psi_{i}^{\varepsilon}\right)_{\varepsilon}$, for a matrix-valued test function $a$ supported in $\omega$,

$$
\begin{aligned}
& \lim _{\varepsilon \rightarrow 0}\left(\operatorname{op}_{\varepsilon}(a) \psi_{j}^{\varepsilon}, \psi_{j}^{\varepsilon}\right)=\operatorname{tr}\left(\int a(x, \xi) d \mu_{j}(x, \xi)\right), \\
& \lim _{\varepsilon \rightarrow 0}\left(\operatorname{op}_{\varepsilon}(a) \psi_{i}^{\varepsilon}, \psi_{j}^{\varepsilon}\right)=\operatorname{tr}\left(\int a(x, \xi) d \mu_{i j}(x, \xi)\right)
\end{aligned}
$$

The first step of the proof consists in proving the following proposition which is a consequence of the analysis performed in Section 6 of [25] and in [26. For the convenience of the reader, we give a proof below in order to emphasize the specific features due to the crossing. We recall that, for all $j$, the set $\Gamma_{j}$ is defined in (1.13) and is included in some codimension one submanifold $\Sigma_{j}$. 
Proposition 3.2. For all $j \in\{1, \cdots, N\}$, there exists a measure $\nu_{j}$ absolutely continuous with respect to $\mu_{j}$ such that, as distributions on $\omega$,

$$
H_{j} \mu_{j}=\left[R_{j}, \mu_{j}\right]+\nu_{j}, \quad \operatorname{Supp} \nu_{j} \subset \Gamma_{j} \cap \omega
$$

where $R_{j}$ is defined in (1.15). Besides, in $\omega$ and outside $\Gamma_{j}, \mu \Pi_{j}=\Pi_{j} \mu=\mu_{j}$.

In the following subsection, we prove Proposition 3.2. Then we study a class of matricial equations containing (3.2) in a second subsection. Finally, the third subsection is devoted to the conclusion of the proof of Theorem 1.6.

\subsection{Proof of Proposition 3.2}

We work microlocally in $\omega$. Let us first prove the commutation relation of $\mu$ with $\Pi_{j}$ outside $\Gamma_{j}$. This is equivalent to the fact that for $k \neq j, \Pi_{k} \mu \Pi_{j}=0$ on $\omega \backslash \Gamma_{j}$, i.e. to

$$
\forall j \in\{1, \cdots, N\}, \forall k \neq j, \quad \forall a \in \mathcal{C}_{0}^{\infty}\left(\omega \backslash \Sigma_{j}, \mathbb{C}^{N, N}\right), \quad\left(\mathrm{op}_{\varepsilon}\left(\Pi_{k} a \Pi_{j}\right) \psi^{\varepsilon}, \psi^{\varepsilon}\right) \underset{\varepsilon \rightarrow 0}{\longrightarrow} 0,
$$

Since $\lambda_{j} \neq \lambda_{k}$ on the support of $a, \frac{1}{\lambda_{k}-\lambda_{j}} \Pi_{k} a \Pi_{j} \in \mathcal{C}_{0}^{\infty}$ and

$$
\Pi_{k} a \Pi_{j}=\left[Q, \frac{1}{\lambda_{k}-\lambda_{j}} \Pi_{k} a \Pi_{j}\right] .
$$

Therefore, (3.3) follows from

$$
\left(\mathrm{op}_{\varepsilon}\left(\Pi_{k} a \Pi_{j}\right) \psi^{\varepsilon}, \psi^{\varepsilon}\right)=\left(\left[\mathrm{op}_{\varepsilon}(Q), \mathrm{op}_{\varepsilon}\left(\frac{1}{\lambda_{k}-\lambda_{j}} \Pi_{k} a \Pi_{j}\right)\right] \psi^{\varepsilon}, \psi^{\varepsilon}\right)+O(\varepsilon)=O(\varepsilon) .
$$

We focus now on the proof of the transport equation (1.16). Since $\lambda_{j}$ may also not belong to a good symbol class, we introduce a function $\tilde{\chi} \in \mathcal{C}_{0}^{\infty}\left(\Omega \times \mathbb{R}^{d} ; \mathbb{R}\right)$ such that $\chi=\tilde{\chi} \chi$. By symbolic calculus,

$$
\begin{aligned}
\mathrm{op}_{\varepsilon}\left(\tilde{\chi} \lambda_{j}\right) \psi_{j}^{\varepsilon}=\mathrm{op}_{\varepsilon}\left(\tilde{\chi} \lambda_{j}\right) \mathrm{op}_{\varepsilon}\left(\chi \Pi_{j}\right) \psi^{\varepsilon} & =\mathrm{op}_{\varepsilon}\left(\tilde{\chi} \lambda_{j} \chi \Pi_{j}\right) \psi^{\varepsilon}+\varepsilon \mathrm{op}_{\varepsilon}\left(\left\{\tilde{\chi} \lambda_{j}, \chi \Pi_{j}\right\} /(2 i)\right) \psi^{\varepsilon}+o(\varepsilon), \\
o(\varepsilon)=\mathrm{op}_{\varepsilon}\left(\chi \Pi_{j}\right) \mathrm{op}_{\varepsilon}(Q) \psi^{\varepsilon} & =\mathrm{op}_{\varepsilon}\left(\chi \Pi_{j} Q\right) \psi^{\varepsilon}+\varepsilon \operatorname{op}_{\varepsilon}\left(\left\{\chi \Pi_{j}, Q\right\} /(2 i)\right) \psi^{\varepsilon}+o(\varepsilon)
\end{aligned}
$$

in $L^{2}$. Since $\chi \Pi_{j} Q=\tilde{\chi} \lambda_{j} \chi \Pi_{j}$, this yields

$$
\mathrm{op}_{\varepsilon}\left(\tilde{\chi} \lambda_{j}\right) \psi_{j}^{\varepsilon}=\frac{\varepsilon}{i} \mathrm{op}_{\varepsilon}\left(\tilde{B}_{j}\right) \psi^{\varepsilon}+o(\varepsilon),
$$

where

$$
\tilde{B}_{j}=\frac{1}{2}\left(-\left\{\chi \Pi_{j}, Q\right\}+\left\{\tilde{\chi} \lambda_{j}, \chi \Pi_{j}\right\}\right)=-\frac{1}{2}\left\{\chi \Pi_{j}, Q+\tilde{\chi} \lambda_{j} \operatorname{Id}\right\} .
$$

Thus, for $a \in C_{0}^{\infty}\left(\omega, \mathbb{C}^{N, N}\right)$,

$$
\begin{aligned}
\frac{i}{\varepsilon}\left(\left[\mathrm{op}_{\varepsilon}(a), \mathrm{op}_{\varepsilon}\left(\tilde{\chi} \lambda_{j}\right)\right] \psi_{j}^{\varepsilon}, \psi_{j}^{\varepsilon}\right) & =\frac{i}{\varepsilon}\left(\mathrm{op}_{\varepsilon}(a) \mathrm{op}_{\varepsilon}\left(\tilde{\chi} \lambda_{j}\right) \psi_{j}^{\varepsilon}, \psi_{j}^{\varepsilon}\right)-\frac{i}{\varepsilon}\left(\mathrm{op}_{\varepsilon}(a) \psi_{j}^{\varepsilon}, \mathrm{op}_{\varepsilon}\left(\tilde{\chi} \lambda_{j}\right) \psi_{j}^{\varepsilon}\right) \\
& =\left(\operatorname{op}_{\varepsilon}(a) \operatorname{op}_{\varepsilon}\left(\tilde{B}_{j}\right) \psi^{\varepsilon}, \psi_{j}^{\varepsilon}\right)+\left(\mathrm{op}_{\varepsilon}(a) \psi_{j}^{\varepsilon}, \mathrm{op}_{\varepsilon}\left(\tilde{B}_{j}\right) \psi^{\varepsilon}\right)+o(1)(3.4) \\
& =\left(\operatorname{op}_{\varepsilon}\left(\Pi_{j} a \tilde{B}_{j}+\tilde{B}_{j}^{*} a \Pi_{j}\right) \psi^{\varepsilon}, \psi^{\varepsilon}\right)+o(1),
\end{aligned}
$$


where $\tilde{B}_{j}^{*}=\frac{1}{2}\left(\left\{Q, \chi \Pi_{j}\right\}+\left\{\tilde{\chi} \lambda_{j}, \chi \Pi_{j}\right\}\right)=\frac{1}{2}\left\{Q+\tilde{\chi} \lambda_{j} \mathrm{Id}, \tilde{\chi} \Pi_{j}\right\}$. On one hand,

$$
\lim _{\varepsilon \rightarrow 0} \frac{i}{\varepsilon}\left(\left[\mathrm{op}_{\varepsilon}(a), \mathrm{op}_{\varepsilon}\left(\tilde{\chi} \lambda_{j}\right)\right] \psi_{j}^{\varepsilon}, \psi_{j}^{\varepsilon}\right)=-\operatorname{tr} \int\left(H_{j} a\right)(x, \xi) d \mu_{j}(x, \xi)=\operatorname{tr} \int a(x, \xi) d\left(H_{j} \mu_{j}\right)(x, \xi),
$$

since $\tilde{\chi}=1$ on the support of $a$, and, on the other hand,

$$
\begin{aligned}
\lim _{\varepsilon \rightarrow 0}\left(\mathrm{op}_{\varepsilon}\left(\Pi_{j} a \tilde{B}_{j}+\tilde{B}_{j}^{*} a \Pi_{j}\right) \psi^{\varepsilon}, \psi^{\varepsilon}\right)=\operatorname{tr}\left(\int\left(\Pi_{j} a \tilde{B}_{j}+\tilde{B}_{j}^{*} a \Pi_{j}\right) d \mu(x, \xi)\right) \\
=\operatorname{tr}\left(\int a\left(\tilde{B}_{j} d \mu(x, \xi) \Pi_{j}+\Pi_{j} d \mu(x, \xi) \tilde{B}_{j}^{*}\right)\right)=\operatorname{tr} \int a d \tilde{\nu}_{j}(x, \xi),
\end{aligned}
$$

where $\tilde{\nu}_{j}=\tilde{B}_{j} \mu \Pi_{j}+\Pi_{j} \mu \tilde{B}_{j}^{*}$. In view of $\chi=1$ on $\bar{\omega}$, we have in $\omega$,

$$
\tilde{\nu}_{j}=B_{j} \mu \Pi_{j}+\Pi_{j} \mu B_{j}^{*}
$$

with $B_{j}$ defined in (1.14). Let us study now $\tilde{\nu}_{j}$. This measure describes the limit of the term (3.4), therefore it is absolutely continuous with respect to the Wigner measure of $\left(\psi_{j}^{\varepsilon}\right)$, namely $\mu_{j}$. Let us focus on $\tilde{\nu}_{j} \mathbf{1}_{\Gamma_{j}^{c}}$, where $\mathbf{1}_{\Gamma_{j}^{c}}$ denotes the characteristic function of the complement $\Gamma_{j}^{c}$ of $\Gamma_{j}$. The computation below is done in [25] and [26]. We write it for the sake of completeness. In view of the commutation relation $\mu \Pi_{j}=\Pi_{j} \mu$ outside $\Gamma_{j}$, we have

$$
\tilde{\nu}_{j} \mathbf{1}_{\Gamma_{j}^{c}}=\left(B_{j} \Pi_{j} \mu+\mu \Pi_{j} B_{j}^{*}\right) \mathbf{1}_{\Gamma_{j}^{c}} .
$$

Besides, writing $Q=\sum_{1 \leq k \leq m} \lambda_{k} \Pi_{k}$, 1.14) implies that

$$
\begin{aligned}
& 2 B_{j}=\left\{\lambda_{j}, \Pi_{j}\right\}-\sum_{1 \leq k \leq m}\left(\lambda_{k}\left\{\Pi_{j}, \Pi_{k}\right\}+\left\{\Pi_{j}, \lambda_{k}\right\} \Pi_{k}\right), \\
& 2 B_{j}^{*}=\left\{\lambda_{j}, \Pi_{j}\right\}+\sum_{1 \leq k \leq m}\left(\lambda_{k}\left\{\Pi_{k}, \Pi_{j}\right\}+\Pi_{k}\left\{\lambda_{k}, \Pi_{j}\right\}\right) .
\end{aligned}
$$

Since $\Pi_{j}^{2}=\Pi_{j}, \Pi_{j}\left\{\lambda_{j}, \Pi_{j}^{2}-\Pi_{j}\right\} \Pi_{j}=0$ and $\Pi_{j}\left\{\lambda_{j}, \Pi_{j}\right\} \Pi_{j}=0$. Therefore

$$
\left(\left\{\lambda_{j}, \Pi_{j}\right\} \Pi_{j} \mu+\mu \Pi_{j}\left\{\lambda_{j}, \Pi_{j}\right\}\right) \mathbf{1}_{\Gamma_{j}^{c}}=\left[\left[\left\{\lambda_{j}, \Pi_{j}\right\}, \Pi_{j}\right], \mu_{j}\right] \mathbf{1}_{\Gamma_{j}^{c}} .
$$

Using the general fact that $A\{B, C\}-\{A, B\} C=\{A B, C\}-\{A, B C\}$, we observe that, $k \neq j$,

$$
\begin{aligned}
\left\{\Pi_{k}, \Pi_{j}\right\} & =\left\{\Pi_{k}^{2}, \Pi_{j}\right\}=\Pi_{k}\left\{\Pi_{k}, \Pi_{j}\right\}-\left\{\Pi_{k}, \Pi_{k}\right\} \Pi_{j}, \\
-\left\{\Pi_{j}, \Pi_{k}\right\} & =-\left\{\Pi_{j}, \Pi_{k}^{2}\right\}=\Pi_{j}\left\{\Pi_{k}, \Pi_{k}\right\}-\left\{\Pi_{j}, \Pi_{k}\right\} \Pi_{k},
\end{aligned}
$$

yielding

$$
-\lambda_{k}\left(\left\{\Pi_{j}, \Pi_{k}\right\} \Pi_{j} \mu-\mu \Pi_{j}\left\{\Pi_{k}, \Pi_{j}\right\}\right) \mathbf{1}_{\Gamma_{j}^{c}}=\lambda_{k}\left[\Pi_{j}\left\{\Pi_{k}, \Pi_{k}\right\} \Pi_{j}, \mu_{j}\right] \mathbf{1}_{\Gamma_{j}^{c}} .
$$

The previous bracket identity with $A=B=C=\Pi_{j}$ implies that $\Pi_{j}\left\{\Pi_{j}, \Pi_{j}\right\}=\left\{\Pi_{j}, \Pi_{j}\right\} \Pi_{j}$, whence

$$
\Pi_{j}\left\{\Pi_{j}, \Pi_{j}\right\}=\Pi_{j}^{2}\left\{\Pi_{j}, \Pi_{j}\right\}=\Pi_{j}\left\{\Pi_{j}, \Pi_{j}\right\} \Pi_{j}, \quad\left\{\Pi_{j}, \Pi_{j}\right\} \Pi_{j}=\left\{\Pi_{j}, \Pi_{j}\right\} \Pi_{j}^{2}=\Pi_{j}\left\{\Pi_{j}, \Pi_{j}\right\} \Pi_{j} .
$$


Replacing both $\Pi_{j}$ 's inside the bracket by Id $-\sum_{k \neq j} \Pi_{k}$ and using (3.10) with $k \neq j$ replaced by $k \neq l$, we obtain

$$
\Pi_{j}\left\{\Pi_{j}, \Pi_{j}\right\} \Pi_{j}=\sum_{k \neq j} \Pi_{j}\left\{\Pi_{k}, \Pi_{k}\right\} \Pi_{j} .
$$

By (3.12) and (3.13),

$$
-\lambda_{j}\left(\left\{\Pi_{j}, \Pi_{j}\right\} \Pi_{j} \mu-\mu \Pi_{j}\left\{\Pi_{j}, \Pi_{j}\right\}\right) \mathbf{1}_{\Gamma_{j}^{c}}=-\lambda_{j} \sum_{k \neq j}\left[\Pi_{j}\left\{\Pi_{k}, \Pi_{k}\right\} \Pi_{j}, \mu_{j}\right] \mathbf{1}_{\Gamma_{j}^{c}} .
$$

Collecting the different pieces (3.9), (3.11) and (3.14), we obtain

$$
2 \tilde{\nu}_{j} \mathbf{1}_{\Gamma_{j}^{c}}=\left[2\left[\left\{\lambda_{j}, \Pi_{j}\right\}, \Pi_{j}\right]+\sum_{k}\left(\lambda_{k}-\lambda_{j}\right) \Pi_{j}\left\{\Pi_{k}, \Pi_{k}\right\} \Pi_{j}, \mu_{j}\right] \mathbf{1}_{\Gamma_{j}^{c}} .
$$

By (3.6) and (3.7), $H_{j} \mu_{j}=\tilde{\nu}_{j}$ that is $H_{j} \mu_{j}=\left[R_{j}, \mu_{j}\right]+\nu_{j}$, where $R_{j}$ is defined by (1.15) and

$$
\nu_{j}=\tilde{\nu}_{j} \mathbf{1}_{\Gamma_{j}}
$$

\subsection{Analysis of a transport equation with a measure valued source term}

We work in a larger setting than those of Theorem 1.6. We consider an open subset $\Omega$ of $\mathbb{R}^{D}(D \geq 1)$, $\Sigma$ a smooth hypersurface of $\Omega$, and

$$
H=\sum_{j=1}^{D} a_{j} \frac{\partial}{\partial x_{j}}=: a \cdot \nabla
$$

a $C^{\infty}$ vector field on $\Omega$ with no singular point (i.e. $\forall x \in \Omega, H(x) \neq 0$ ) and with real valued coefficients $a_{j}$. Let $\tilde{x} \in \Sigma$ and $\omega$ be an open neighborhood of $\tilde{x}$ in $\Omega$ such that $\Sigma \cap \omega=\{\gamma=0\}$, where $\gamma$ is smooth on $\omega$ and $\nabla \gamma$ does not vanish on $\Sigma \cap \omega$. We start with a few definitions.

- $\tilde{x} \in \Sigma(0 ; H)$ if and only if $H$ is transverse to $\Sigma$ at $\tilde{x}$, that is $H \gamma(\tilde{x}) \neq 0$.

- $\tilde{x} \in \Sigma(k ; H), k \in \mathbb{N}^{*}$ if and only if $H \gamma(\tilde{x})=\ldots=H^{k} \gamma(\tilde{x})=0$ and $H^{k+1} \gamma(\tilde{x}) \neq 0$.

- $\tilde{x} \in \Sigma(\infty ; H)$ if and only if $H^{k}(\tilde{x})=0$ for all $k \in \mathbb{N}$.

In the two first cases, we say that $\Sigma$ has a finite order contact with $H$. In the sequel we shall need some notions and results of set theory, in particular the notions of ordinals and of transfinite induction. We refer to [34, 35, 48] for details.

Definition 3.3. Define by transfinite recursion the set $F(\alpha ; H ; \Sigma)$, where $\alpha$ is an ordinal, by:

- $F(0 ; H ; \Sigma)=\Sigma(\infty ; H)$.

- If $\alpha=\beta+1$ is a successor ordinal, $F(\alpha ; H ; \Sigma)$ is obtained by taking off from $F(\beta ; H ; \Sigma)$ the open subset of points $\tilde{x} \in F(\beta ; H ; \Sigma)$ for which there exists an open neighborhood $U$ of $\tilde{x}$ and an hypersurface $\Theta$ such that $(U \cap F(\beta ; H ; \Sigma)) \subset(U \cap \Theta)$ and such that $H$ has only contact of finite order with $\Theta$ at $\tilde{x}$ (i.e. $\tilde{x} \in \Theta(k ; H)$ for some $k \in \mathbb{N}$ ).

- If $\alpha=\bigcup_{\beta<\alpha} \beta$ is a limit ordinal, then $F(\alpha ; H ; \Sigma)=\bigcap_{\beta<\alpha} F(\beta ; H ; \Sigma)$. 
Notice that $F(0 ; H ; \Sigma)$, and thus any $F(\alpha ; H ; \Sigma)$, is a closed subset of $\Omega$. Furthermore, $F(\cdot ; H ; \Sigma)$ is non increasing: $F(\beta ; H ; \Sigma) \subset F(\alpha ; H ; \Sigma)$ if $\beta>\alpha$. The family of all $F(\alpha ; H ; \Sigma)$ is a family of closed subsets of $\mathbb{R}^{D}$ which is well-ordered for the inclusion. Using that $\mathbb{R}^{D}$ has a countable basis consisting of open subsets one may show that this family is countable, and thus may be indexed by a countable ordinal. As a consequence, there exists a (countable) ordinal $\beta_{0}$ such that $F(\alpha ; H ; \Sigma)=F\left(\beta_{0} ; H ; \Sigma\right)$, for all $\alpha>\beta_{0}$.

Definition 3.4. We denote by $\mathcal{F}(H ; \Sigma)=F\left(\beta_{0} ; H ; \Sigma\right)$. In the context of Theorems 1.3 and 1.6, we will write, for $j=1, \ldots, m, \mathcal{F}_{j}=\mathcal{F}\left(H_{j} ; \Sigma_{j}\right)$, where $\Sigma_{j}$ is an hypersurface containing the eigenvalues crossing and $H_{j}$ is the Hamiltonian flow associated to the function $\lambda_{j}$.

In this section, we prove

Proposition 3.5. Let $\mu$ be a matrix-valued Radon measure on $\Omega$, such that, as distributions,

$$
H \mu+b \mu+\mu c=\nu,
$$

where $b$ and $c$ are smooth matrix-valued functions on $\Omega$ and $\nu$ is a matrix-valued Borel measure on $\Omega$ absolutely continuous w.r.t. $\mu$. Assume furthermore that

$$
\operatorname{supp} \nu \subset \Sigma \text {. }
$$

Then $\operatorname{supp} \nu \subset \mathcal{F}(H ; \Sigma)$.

Remark 3.6. Proposition 3.5 implies that, in the sense of distributions on the open set $\Omega \backslash \mathcal{F}(H ; \Sigma)$,

$$
H \mu+b \mu+\mu c=0 .
$$

The proof of Proposition 3.5 proceeds in three steps. In Lemma 3.7, we prove that $\mathbf{1}_{\Sigma(0 ; H)} \nu=0$, then, in Lemma 3.8, that $\mathbf{1}_{\Sigma(k ; H)} \nu=0$ for all $k \in \mathbb{N}$. These two facts imply that $\operatorname{Supp} \nu \subset F(0 ; H ; \Sigma)$ and we can conclude the proof by transfinite induction.

Lemma 3.7. Let $H, b, c$ and $\Sigma$ be as in Proposition 3.5. Then $\mathbf{1}_{\Sigma(0 ; H)} \mu=\mathbf{1}_{\Sigma(0 ; H)} \nu=0$.

\section{Proof}

This is a classical property; we give a proof for the sake of completeness.

Since $\nu$ is absolutely continuous w.r.t. $\mu$, it suffices to show $\mathbf{1}_{\Sigma(0 ; H)} \mu=0$. We must show a local property, namely that if $\tilde{x} \in \Sigma(0 ; H)$, there exists an open neighborhood $U$ of $\tilde{x}$ such that $\mathbf{1}_{U \cap \Sigma} \mu=0$. Let $\tilde{x} \in \Sigma(0 ; H)$.

Step 1. Reduction of the problem. We first show that we may assume (locally) that $H=\frac{\partial}{\partial x_{1}}$, i.e. that we may assume that near $\tilde{x}, \mu$ satisfies an equation of the form

$$
\frac{\partial}{\partial x_{1}} \mu+b \mu+\mu c=\nu
$$

Consider a neighborhood $U$ of $\tilde{x}$ such that $\Sigma$ is defined, in $U$, by the equation $\gamma=0$, where $\gamma$ is a $C^{\infty}$ function such that $\nabla \gamma(x) \neq 0$ for all $x$ in $U$. Using that $H$ is transverse to $\Sigma$ in $\tilde{x}$, and taking a smaller neighborhood $U$ of $\tilde{x}$ if necessary, one may find a $C^{\infty}$ diffeomorphism $\chi$ from an open subset $V$ of $\mathbb{R}_{y}^{D}$ to $U$ such that

$$
\forall f \in C_{0}^{\infty}(U), \quad \frac{\partial}{\partial y_{1}}(f \circ \chi)=(H f) \circ \chi .
$$


Define the measures $\mu_{1}$ and $\nu_{1}$ in $V$ by

$$
\forall f \in C_{c}^{0}(U), \quad\left\langle\mu_{1}, f \circ \chi\right\rangle=\langle\mu, f\rangle, \quad\left\langle\nu_{1}, f \circ \chi\right\rangle=\langle\nu, f\rangle .
$$

By $(3.17)$ and $(3.16)$, for $f \in C_{c}^{0}(U)$,

$$
\begin{aligned}
\left\langle\frac{\partial}{\partial y_{1}} \mu_{1}, f \circ \chi\right\rangle & =-\left\langle\mu_{1}, \frac{\partial}{\partial y_{1}}(f \circ \chi)\right\rangle=-\left\langle\mu_{1},(H f) \circ \chi\right\rangle=-\langle\mu, H f\rangle \\
& =\langle H \mu, f\rangle+\langle(\nabla \cdot a) \mu, f\rangle \\
& =\langle\nu, f\rangle-\langle\mu, b f\rangle-\langle\mu, f c\rangle+\langle\mu,(\nabla \cdot a) f\rangle \\
& =\left\langle\nu_{1}, f \circ \chi\right\rangle-\left\langle(b \circ \chi-(\nabla \cdot a) \circ \chi) \mu_{1}, f \circ \chi\right\rangle-\left\langle\mu_{1}(c \circ \chi), f \circ \chi\right\rangle .
\end{aligned}
$$

Thus $\left(\partial / \partial y_{1}\right) \mu_{1}+b_{1} \mu_{1}+\mu_{1} c_{1}=\nu_{1}$ in $V$, with smooth $b_{1}:=b \circ \chi-(\nabla \cdot a) \circ \chi$ and $c_{1}:=c \circ \chi$.

Step 2. Proof of the vanishing of $\mathbf{1}_{\Sigma} \mu$ near $\tilde{x}$. We now assume that (3.19) holds near $\tilde{x} \in \Sigma(0 ; H)$, where $H=\frac{\partial}{\partial x_{1}}$. Of course one may assume $\tilde{x}=0$. Let $U$ a small open neighborhood of 0 such that $\Sigma \cap U=\{\gamma(x)=0\}$ where $\gamma$ is real and smooth, and $\partial_{x_{1}} \gamma \geq c_{0}>0$ on $U$. Consider $\chi \in \mathcal{C}_{0}^{\infty}(\mathbb{R} ; \mathbb{R})$ a bounded function such that $\chi^{\prime}(0)=1$. Let $\psi \in \mathcal{C}_{0}^{\infty}(U)$ with matrix values. Then $\phi:=\left(1 / \partial_{x_{1}} \gamma\right) \psi \in \mathcal{C}_{0}^{\infty}(U)$. By (3.19), for all $\varepsilon>0$,

$$
\left\langle\partial_{x_{1}} \mu, \chi(\gamma / \varepsilon) \phi\right\rangle=\langle\nu, \chi(\gamma / \varepsilon) \phi\rangle-\langle\mu, \chi(\gamma / \varepsilon) b \phi\rangle-\langle\mu, \chi(\gamma / \varepsilon) \phi c\rangle=O\left(\varepsilon^{0}\right) .
$$

Therefore

$$
O\left(\varepsilon^{0}\right)=\left\langle\partial_{x_{1}} \mu, \chi(\gamma / \varepsilon) \phi\right\rangle=-\left\langle\mu, \chi(\gamma / \varepsilon) \partial_{x_{1}} \phi\right\rangle-\frac{1}{\varepsilon}\left\langle\mu, \chi^{\prime}(\gamma / \varepsilon)\left(\partial_{x_{1}} \gamma\right) \phi\right\rangle=O\left(\varepsilon^{0}\right) .
$$

Multiplying by $\varepsilon$ and letting $\varepsilon$ go to 0 , we arrive at: $\left\langle\mu,\left(\partial_{x_{1}} \gamma\right) \mathbf{1}_{\Sigma} \phi\right\rangle=0$. Thus $\left\langle\mu, \mathbf{1}_{\Sigma} \psi\right\rangle=0$ and $\mathbf{1}_{\Sigma} \mu$ vanishes on $U$.

We next show that we can extend the preceding Lemma to finite order tangency.

Lemma 3.8. Let $H, b, c$ and $\Sigma$ be as in Proposition 3.5. For all $k \geq 0, \mathbf{1}_{\Sigma(k ; H)} \mu=\mathbf{1}_{\Sigma(k ; H)} \nu=0$.

\section{Proof}

We will use, in a simpler setting, an induction argument on the order at which $H$ is tangent to $\Sigma$ which is related to the argument in [39, Section 4] (see also a similar argument in the context of defect measures in [5]).

As $\nu$ is absolutely continuous w.r.t. $\mu$, it is sufficient to show $\mathbf{1}_{\Sigma(k ; H)} \mu=0$, for all $k \geq 0$. Let us prove this assertion by induction. By Lemma 3.7, the property holds for $k=0$. Assume that, for some $k \geq 0$,

$$
\mathbf{1}_{\Sigma(0 ; H)} \mu=\mathbf{1}_{\Sigma(1 ; H)} \mu=\ldots=\mathbf{1}_{\Sigma(k ; H)} \mu=0 .
$$

Let $\tilde{x} \in \Sigma(k+1 ; H)$. By the definition of $\Sigma(k+1 ; H), H^{k+1} \gamma(\tilde{x})=0$ and $H^{k+2} \gamma(\tilde{x}) \neq 0$. Let $U$ be a small neighborhood of $\tilde{x}$ such that

$$
\forall x \in U, \quad H^{k+2} \gamma(x) \neq 0 .
$$

By (3.21), the set

$$
\Xi:=\left\{x \in U, H^{k+1} \gamma(x)=0\right\}
$$

is an hypersurface and $H$ is transverse to $\Xi$. The support of $\nu$ is included in $\Sigma=\bigcup_{0 \leq j \leq \infty} \Sigma(j ; H)$. Furthermore, $\nu$ is absolutely continuous with respect to $\mu$, thus (3.20) implies that $\operatorname{supp} \nu \subset$ 
$\bigcup_{k+1 \leq j \leq \infty} \Sigma(j ; H)$. In particular, supp $\nu_{\uparrow U} \subset \Xi$. By Lemma 3.7 with $\Sigma$ replaced by $\Xi$ and $\Omega$ replaced by $U$,

$$
\mathbf{1}_{\Sigma(k+1 ; H) \cap U} \mu=\mathbf{1}_{\left\{x \in U \mid H^{j+1} \gamma(x)=0, H^{j+2} \gamma(x) \neq 0\right\}} \mu=0,
$$

which completes the induction argument.

We can now close the proof of Proposition 3.5.

\section{Proof}

We will show by transfinite induction that for all ordinal $\alpha, \operatorname{supp} \nu \subset F(\alpha ; H ; \Sigma)$. For simplicity, denote $F(\alpha ; H ; \Sigma)$ by $F(\alpha)$. By Lemma 3.8, $\nu$ vanishes on $\Sigma(k ; H)=0$, for all $k$. Using that $\operatorname{supp} \nu \subset \Sigma$ and that

$$
\Sigma \backslash \bigcup_{0 \leq k<\infty} \Sigma(k ; H)=\Sigma(\infty ; H)=F(0)
$$

is a closed set, we get that $\operatorname{supp} \nu \subset F(0)$.

Let us take an ordinal $\alpha>0$ and assume that $\operatorname{supp} \nu \subset F(\beta)$ for all $\beta<\alpha$. If $\alpha$ is a limit ordinal, then by the induction hypothesis $\operatorname{supp} \nu \subset \bigcap_{\beta<\alpha} F(\beta)=F(\alpha)$.

If $\alpha=\beta+1$ for some $\beta$, then we know by the induction hypothesis that $\operatorname{supp} \nu \subset F(\beta)$. Let $x \in F(\beta) \backslash F(\alpha)$. This means that there exists an open neighborhood $U$ of $x$ in $\Omega$ such that $F(\beta) \cap U \subset \Theta$, where $\Theta$ is a codimension 1 submanifold of $U$ which has only finite order contact with $H$. By induction hypothesis, supp $\nu_{\uparrow U} \subset \Theta$. By Lemma 3.8, $\mathbf{1}_{\Theta} \nu_{\uparrow U}=0$, which shows that $x \notin \operatorname{supp} \nu$. Thus $\operatorname{supp} \nu \subset F(\alpha)$, which ends the proof of Proposition 3.5.

Let us finally have a closer look at the set $\mathcal{F}(H ; \Sigma)$. We first notice that if $\Sigma$ contains a piece $(x(s))_{s \in\left(0, s_{0}\right)}$ of a characteristic curve of the field $H$, then so does $\Sigma(\alpha ; H)$ for all ordinal $\alpha$, and thus $\mathcal{F}(H ; \Sigma)$ is not empty. A sufficient condition for $\mathcal{F}(H ; \Sigma)$ to be empty is given by the following proposition, which we will prove by a classical argument using Baire's Theorem.

Proposition 3.9. Let $\Sigma$ be a codimension 1 submanifold in an open subset $\Omega$ of $\mathbb{R}^{D}$ and $H$ a smooth vector field in $\Omega$. Assume

$$
\Sigma(\infty ; H) \subset \bigcup_{j \in J} Z_{j}
$$

where $\left\{Z_{j}\right\}_{j \in J}$ is a finite or countable family of hypersurfaces that have only a finite order contact with $H$. Then $\mathcal{F}(H ; \Sigma)=\emptyset$.

In particular, if $\Sigma(\infty ; H)$ is at most countable or is an hypersurface with only finite order contact with $H$, then $\mathcal{F}(H ; \Sigma)$ is empty.

\section{Proof}

Denote again $F(\alpha ; H ; \Sigma)$ by $F(\alpha)$ for simplicity. We will show by induction on the ordinal $\alpha$ that the property $\mathcal{P}(\alpha)$ :

"For all ordinal $\beta$ such that $\beta<\alpha$ and $F(\beta) \neq \emptyset, F(\alpha)$ is strictly included in $F(\beta)$."

holds for all ordinal $\alpha$. It is classical that this implies that $F(\alpha)$ is empty if $\alpha$ is large enough.

The property $\mathcal{P}(0)$ holds trivially.

Let $\alpha>0$ be an ordinal and assume that $\mathcal{P}(\gamma)$ holds for any $\gamma<\alpha$. First assume that $\alpha$ is a limit ordinal. Let $\beta<\alpha$ and assume that $F(\beta)$ is not empty. Then $\beta+1<\alpha$, and the property $\mathcal{P}(\beta+1)$ implies that $F(\beta+1)$ is strictly included in $F(\beta)$. As a consequence, $F(\alpha)$ is strictly included in $F(\beta)$, which shows $\mathcal{P}(\alpha)$. 
It remains to treat the case when $\alpha=\beta+1$ is a successor ordinal. Assume that $F(\alpha)=F(\beta)$. Using assumption (3.22) and that $F(\beta) \subset F(\alpha)$, we get

$$
F(\beta)=\bigcup_{j \in J}\left(F(\beta) \cap Z_{j}\right)
$$

The sets $F(\beta) \cap Z_{j}$ are closed in $F(\beta)$. We will show that they have empty interior in $F(\beta)$. If not, there would exist an open subset $U$ of $\Omega$ such that $\emptyset \neq F(\beta) \cap U \subset F(\beta) \cap Z_{j}$. As $Z_{j}$ is an hypersurface which have only finite order contact with $H$, we would get by the definition of $F(\alpha)=F(\beta+1)$ that $F(\alpha) \cap U$ is empty, contradicting the fact that $F(\alpha)=F(\beta)$.

The union (3.23) is thus a countable union of closed subsets of $F(\beta)$ that have empty interior in $F(\beta)$. By Baire Theorem in the complete metric space $F(\beta)$, we get that $F(\beta)$ has empty interior in itself, which shows of course that $F(\beta)$ is empty, concluding the proof of the induction argument and thus of Proposition 3.9.

\subsection{Proof of the propagation}

Let us now conclude the proof of Theorem 1.6. Recall that $\mu_{j}=\Pi_{j} \mu \Pi_{j}$ on $\omega$ (see before (3.1)). By Proposition 3.2, $H_{j} \mu_{j}=\left[R_{j}, \mu_{j}\right]+\nu_{j}$ on $\omega$, where $\nu_{j}$ is given by (3.15) and (3.8), and $\operatorname{supp} \nu_{j} \subset$ $\Gamma_{j} \cap \omega$. Since $\Gamma_{j}$ is included in a codimension one submanifold $\Sigma_{j}$, Proposition 3.5 shows that $\operatorname{supp} \nu_{j} \subset \omega \cap \mathcal{F}_{j}$, where $\mathcal{F}_{j}=\mathcal{F}\left(H_{j} ; \Sigma_{j}\right)$ (cf. Definition 3.4). Since the matrix $B_{j}$ vanishes on $\omega \cap \mathcal{F}_{j}$, by assumption, so does also $\nu_{j}$. Thus $\nu_{j}=0$. This closes the proof of Theorem 1.6.

\section{Highly degenerated situations}

In this section, we give an example of $2 \times 2$-matrix valued potential $M$ as in (1.4) for which the assumptions of Theorem 1.1 are not satisfied. Furthermore the conclusion of Theorem 1.6 (and therefore its hypothesis) is also false in that case.

The potential: We choose $d=N=2$ and take $d_{1}: \mathbb{R} \rightarrow \mathbb{R}$ a smooth function such that $d_{1}^{\prime}(0)>0$. Take $\chi: \mathbb{R} \rightarrow \mathbb{R}$ a smooth function such that $\chi=0$ on $[0 ; 1]$ and positive outside. Let

$$
d\left(x_{1}, x_{2}\right)=\chi\left(x_{2}\right) d_{1}\left(x_{1}\right)
$$

and, for some $k \in \mathbb{R}$,

$$
M(x)=d(x) \operatorname{Id}+\exp \left(-\frac{1}{\left|x_{1}\right|}\right) V\left(x_{2}\right) \text { where } V\left(x_{2}\right)=\left(\begin{array}{cc}
\cos \left(2 k \pi x_{2}\right) & \sin \left(2 k \pi x_{2}\right) \\
\sin \left(2 k \pi x_{2}\right) & -\cos \left(2 k \pi x_{2}\right)
\end{array}\right) .
$$

The crossing set is $\mathcal{C}=\left\{x_{1}=0\right\}$. We denote by $\lambda^{ \pm}(x), \Pi^{ \pm}\left(x_{2}\right)$ the eigenvalues and eigenprojectors associated with $M$ :

$$
\begin{gathered}
\lambda^{ \pm}(x)=\chi\left(x_{2}\right) d_{1}\left(x_{1}\right) \pm \exp \left(-\frac{1}{\left|x_{1}\right|}\right) \\
\Pi^{+}\left(x_{2}\right)=\frac{1}{2}\left(\begin{array}{cc}
2 \cos ^{2}\left(k \pi x_{2}\right) & \sin \left(2 k \pi x_{2}\right) \\
\sin \left(2 k \pi x_{2}\right) & 2 \sin ^{2}\left(k \pi x_{2}\right)
\end{array}\right), \Pi^{-}\left(x_{2}\right)=\frac{1}{2}\left(\begin{array}{cc}
2 \sin ^{2}\left(k \pi x_{2}\right) & -\sin \left(2 k \pi x_{2}\right) \\
-\sin \left(2 k \pi x_{2}\right) & 2 \cos ^{2}\left(k \pi x_{2}\right)
\end{array}\right) .
\end{gathered}
$$


We are concerned with the time-dependent semiclassical Schrödinger equation

$$
i \varepsilon \partial_{t} \psi^{\varepsilon}=-\frac{\varepsilon^{2}}{2} \Delta \psi^{\varepsilon}+M(x) \psi^{\varepsilon}
$$

with initial data $\psi_{0}^{\varepsilon}$, such that $\left(\psi_{0}^{\varepsilon}\right)_{\varepsilon>0}$ is bounded in $L^{2}\left(\mathbb{R}^{2}\right)$.

The classical trajectories: The trajectories $s \mapsto\left(x_{1}^{ \pm}(s), x_{2}^{ \pm}(s), \xi_{1}^{ \pm}(s), \xi_{2}^{ \pm}(s)\right)$ satisfy

$$
\begin{gathered}
\dot{x}_{1}^{ \pm}(s)=\xi_{1}^{ \pm}(s), \quad \dot{\xi}_{1}^{ \pm}(s)=-\chi\left(x_{2}^{ \pm}(s)\right) d_{1}^{\prime}\left(x_{1}^{ \pm}(s)\right) \mp \frac{x_{1}^{ \pm}(s)}{\left|x_{1}^{ \pm}(s)\right|^{3}} \mathrm{e}^{-1 /\left|x_{1}^{ \pm}(s)\right|} \\
\dot{x}_{2}^{ \pm}(s)=\xi_{2}^{ \pm}(s), \quad \dot{\xi}_{2}^{ \pm}(s)=-\chi^{\prime}\left(x_{2}^{ \pm}(s)\right) d_{1}\left(x_{1}^{ \pm}(s)\right),
\end{gathered}
$$

with the convention that $\exp (-1 /|t|) t /|t|^{3}=0$ for $t=0$. We choose the initial condition: $x_{1}^{ \pm}(0)=$ $\xi_{1}^{ \pm}(0)=x_{2}^{ \pm}(0)=0$ and $\xi_{2}^{ \pm}(0)=\eta>0$. We see that, on $[0,1 / \eta], x_{1}^{ \pm}(s)=0=\xi_{1}^{ \pm}(s), \xi_{2}^{ \pm}(s)=\eta$, and $x_{2}^{ \pm}(s)=s \eta$. Thus, on this time interval, the + and - trajectories coincide and stay in the crossing set $\left\{(x ; \xi) \in\left(\mathbb{R}^{2}\right)^{2} ; x_{1}=0\right\}$.

The data: We choose as initial data a family $\left(\psi_{0}^{\varepsilon}\right)_{\varepsilon>0}$ having a unique Wigner measure of the form

$$
\mu(x, \xi)=\delta\left(x_{1}\right) \otimes \delta\left(x_{2}\right) \otimes \delta\left(\xi_{1}\right) \otimes \delta\left(\xi_{2}-\eta\right)\left(a^{+} \Pi^{+}(0)+a^{-} \Pi^{-}(0)\right)
$$

with $a^{ \pm} \in \mathbb{R}^{+}$. The later is localized at the previously chosen initial condition for the trajectories. We expect that the behaviour of the solutions of (4.2) depends on the concentration around the crossing $\mathcal{C}$ of $\left(\psi_{0}^{\varepsilon}\right)_{\varepsilon>0}$. To describe this concentration, we introduce a two-scale Wigner measures.

We choose $\alpha \in] 0,1 / 2[$ and we analyze

$$
I^{\varepsilon}(a)=\left(\operatorname{op}_{\varepsilon}\left(a\left(x, \xi, \frac{x_{1}}{\varepsilon^{\alpha}}\right)\right) \psi_{0}^{\varepsilon}, \psi_{0}^{\varepsilon}\right)
$$

for symbols $a \in \mathcal{C}^{\infty}\left(\mathbb{R}^{5}\right)$ satisfying

- there exists a compact $K$ of $\mathbb{R}^{4}$ such that, for all $y \in \mathbb{R}, a(\cdot, \cdot, y) \in \mathcal{C}_{0}^{\infty}(K)$,

- there exists $R_{0}>0$ and two smooth functions $a(x, \xi,+\infty)$ and $a(x, \xi,-\infty)$ such that for $|y|>R_{0}, a(x, \xi, y)=a(x, \xi, \operatorname{sgn}(y) \infty)$.

According to [40] (see also [16]), a two-scale Wigner measure associated to the concentration of $\left(\psi_{0}^{\varepsilon}\right)_{\varepsilon>0}$ on $\left\{x_{1}=0\right\}$ at the scale $\varepsilon^{\alpha}$ is a nonnegative Radon measure $\nu$ on $\mathbb{R}^{4} \times \overline{\mathbb{R}}$ where $\overline{\mathbb{R}}=$ $(\mathbb{R} \cup\{-\infty,+\infty\})$ such that, for some sequence $\left(\varepsilon_{k}\right)_{k}$ tending to $0, \lim _{k} I^{\varepsilon_{k}}(a)=\langle a, \nu\rangle$. One then recovers $\mu$ by projection of $\nu$ on $\mathbb{R}^{2 d}$ through

$$
\mu(x, \xi)=\int_{\mathbb{R}} \nu(x, \xi, d y)
$$

and one can decompose $\nu$ as

$$
\nu(x, \xi, y)=\delta\left(x_{1}\right) \otimes\left(\gamma\left(x_{2}, \xi, y\right) \mathbf{1}_{|y|<\infty}+\gamma_{\infty}\left(x_{2}, \xi, y\right) \mathbf{1}_{|y|=\infty}\right)+\mu(x, \xi) \otimes \delta\left(y-\frac{x_{1}}{\left|x_{1}\right|} \infty\right) \mathbf{1}_{\left\{x_{1} \neq 0\right\}} .
$$

We choose $\left(\psi_{0}^{\varepsilon}\right)_{\varepsilon>0}$ such that its concentration above $\left\{(x ; \xi) \in\left(\mathbb{R}^{2}\right)^{2} ; x_{1}=0\right\}$ at the scale $\varepsilon^{\alpha}$ is given by a unique two-scale Wigner measure

$$
\nu(x, \xi, y)=\mathbf{1}_{|y|<+\infty} \delta\left(x_{1}\right) \otimes \delta\left(x_{2}\right) \otimes \delta\left(\xi_{1}\right) \otimes \delta\left(\xi_{2}-\eta\right)\left(\gamma^{+}(y) \Pi^{+}(0)+\gamma^{-}(y) \Pi^{-}(0)\right)
$$


where $\gamma^{ \pm}$are finite nonnegative Radon measures on $\mathbb{R}$. Of course, one has $a^{ \pm}=\int_{\mathbb{R}} \gamma^{ \pm}(d y)$. We emphasize that the two-scale Wigner measure $\nu$ is supported on $\mathbb{R}^{4} \times \mathbb{R}$ so that $\nu(\{-\infty,+\infty\})=0$. The following proposition describes the Wigner measure of $\psi^{\varepsilon}(t)$ for $t \in[0,1 / \eta]$.

Proposition 4.1. There exists a sequence $\left(\varepsilon_{k}\right)_{k}$ tending to 0 and a family of nonnegative Radon measure $\mu_{t}$ such that for all $t \in[0,1 / \eta]$ and for all $a \in \mathcal{C}_{0}^{\infty}\left(\mathbb{R}^{2 d}, \mathbb{C}^{2,2}\right)$,

$$
\left(\mathrm{op}_{\varepsilon_{k}}(a) \psi^{\varepsilon_{k}}(t), \psi^{\varepsilon_{k}}(t)\right) \underset{k \rightarrow+\infty}{\longrightarrow} \operatorname{tr} \int_{\mathbb{R}^{2 d}} a(x, \xi) \mathrm{d} \mu_{t}(\mathrm{~d} x, \mathrm{~d} \xi) .
$$

Besides,

$$
\mu_{t}(x, \xi)=\delta\left(x_{1}\right) \otimes \delta\left(x_{2}-t \eta\right) \otimes \delta\left(\xi_{1}\right) \otimes \delta\left(\xi_{2}-\eta\right)\left(a^{+} \Pi^{+}(0)+a^{-} \Pi^{-}(0)\right) .
$$

Remark 4.2. Let $\Omega=\left\{x \in \mathbb{R}^{2} ;|x|<2\right\}$ and $\theta \in \mathcal{C}_{0}^{\infty}\left(\mathbb{R}^{2}\right)$ such that $\theta=1$ near $\Omega$. The family $\left(\psi^{\varepsilon}\right)_{\varepsilon>0}$ satisfies (1.12) with $Q(x ; \xi)=\left(|\xi|^{2} / 2+d(x)\right) \operatorname{Id}+\theta(x) M(x)$. Except the vanishing condition on the $B_{j}$, the assumptions of Theorem 1.6 are satisfied.

Remark 4.3. We deduce from (4.7) that the measures $\mu_{t}^{ \pm}=\Pi^{ \pm} \mu_{t} \Pi^{ \pm}$satisfy

$$
\mu_{t}^{ \pm}(x, \xi)=\delta\left(x_{1}\right) \otimes \delta\left(x_{2}-t \eta\right) \otimes \delta\left(\xi_{1}\right) \otimes \delta\left(\xi_{2}-\eta\right) \Pi^{ \pm}(t \eta)\left(a^{+} \Pi^{+}(0)+a^{-} \Pi^{-}(0)\right) \Pi^{ \pm}(t \eta) .
$$

We observe that, depending on the choice of $k$, it is possible to have energy transfers between the modes. More precisely, in view of (4.1), we have the following facts:

- For $k \in \mathbb{Z}+1 / 2$, between $t=0$ and $t=1 / \eta$, the mass $a^{+}$is transfered from the plus mode to the minus one. The conclusion of Theorem 1.6 is not true.

- For $k \notin \mathbb{Z} \cup(\mathbb{Z}+1 / 2)$, between $t=0$ and $t=1 / \eta$, there is some partial transfer from one mode to the other. The conclusion of Theorem 1.6 is false.

- For $k=0$, the conclusion of Theorem 1.6 holds true since the $B_{ \pm}$defined in (1.14) vanish on the crossing.

\section{Proof}

The proof of this proposition relies on the analysis of the two-scale Wigner measure associated with $\psi^{\varepsilon}(t)$ which is given by the following lemma.

Lemma 4.4. There exists a sequence $\left(\varepsilon_{k}\right)$ and a family of nonnegative Radon measure $\nu_{t}$ such that for all $t \in[0,1 / \eta]$ and for all $a \in \mathcal{C}_{0}^{\infty}\left(\mathbb{R}^{2 d+1}, \mathbb{C}^{2,2}\right)$,

$$
\left(\operatorname{op}_{\varepsilon_{k}}\left(a\left(x, \xi, \frac{x_{1}}{\varepsilon^{\alpha}}\right)\right) \psi^{\varepsilon_{k}}(t), \psi^{\varepsilon_{k}}(t)\right) \underset{k \rightarrow+\infty}{\longrightarrow} \operatorname{tr} \int_{\mathbb{R}^{2 d} \times \mathbb{R}} a(x, \xi, y) \mathrm{d} \nu_{t}(\mathrm{~d} x, \mathrm{~d} \xi, \mathrm{d} y) .
$$

Besides, $t \mapsto \nu_{t}$ is continuous and $\nu_{t}$ satisfies the transport equation

$$
\partial_{t} \nu_{t}+\xi \cdot \nabla_{x} \nu_{t}-\nabla_{x} d \cdot \nabla_{\xi} \nu_{t}=0
$$

with initial condition $\nu_{0}=\nu$, given by (4.5).

Solving the transport equation (4.8), we obtain

$$
\nu_{t}(x, \xi, y)=\delta\left(x_{1}\right) \otimes \delta\left(x_{2}-t \eta\right) \otimes \delta\left(\xi_{1}\right) \otimes \delta\left(\xi_{2}-\eta\right)\left(\gamma^{+}(y) \Pi^{+}(0)+\gamma^{-}(y) \Pi^{-}(0)\right) .
$$

For each $t \in[0,1 / \eta]$, the sequence $\left(\psi^{\varepsilon_{k}}(t)\right)_{k}$ has a unique Wigner measure $\mu_{t}$ given by (4.4) with $\mu, \nu$ replaced by $\mu_{t}, \nu_{t}$ respectively. This yields (4.6) and 4.7). 
Let us now prove the Lemma.

Proof

Consider a symbol $a \in \mathcal{C}_{0}^{\infty}\left(\mathbb{R}^{2 d+1}, \mathbb{C}^{2,2}\right)$ and let us analyze the quantity

$$
I_{a}^{\varepsilon}(t)=\left(\mathrm{op}_{\varepsilon}\left(a\left(x, \xi, \frac{x_{1}}{\varepsilon^{\alpha}}\right)\right) \psi^{\varepsilon}(t), \psi^{\varepsilon}(t)\right) .
$$

By (4.2),

$$
\frac{\mathrm{d}}{\mathrm{d} t} I_{\varepsilon, a}(t)=\left(K_{\varepsilon} \psi^{\varepsilon}(t), \psi^{\varepsilon}(t)\right) \text { with } K_{\varepsilon}=\frac{1}{i \varepsilon}\left[\operatorname{op}_{\varepsilon}\left(a\left(x, \xi, \frac{x_{1}}{\varepsilon^{\alpha}}\right)\right),-\frac{\varepsilon^{2}}{2} \Delta+M(x)\right] .
$$

We use the scaling operator $T$ defined by

$$
\forall f \in L^{2}\left(\mathbb{R}^{2}\right), \quad T f(x)=\varepsilon^{\alpha / 2} f\left(x_{1} \varepsilon^{\alpha}, x_{2}\right) .
$$

It is unitary on $L^{2}\left(\mathbb{R}^{2}\right)$. In $\mathcal{L}\left(L^{2}\left(\mathbb{R}^{d}\right)\right), K_{\varepsilon}$ can be written as

$$
\begin{gathered}
\frac{T^{*}}{i \varepsilon}\left[\mathrm{op}_{1}\left(a\left(x_{1} \varepsilon^{\alpha}, x_{2}, \xi_{1} \varepsilon^{1-\alpha}, \xi_{2} \varepsilon, x_{1}\right)\right),-\frac{\varepsilon^{2(1-\alpha)}}{2} \partial_{x_{1}}^{2}-\frac{\varepsilon^{2}}{2} \partial_{x_{2}}^{2}+d\left(x_{1} \varepsilon^{\alpha}, x_{2}\right)+\mathrm{e}^{-1 /\left(\varepsilon^{\alpha}\left|x_{1}\right|\right)} V\left(x_{2}\right)\right] T \\
=\begin{array}{c}
\mathrm{op}_{\varepsilon}\left(\xi \cdot\left(\partial_{x} a\right)\left(x, \xi, x_{1} \varepsilon^{-\alpha}\right)-\nabla d(x) \cdot\left(\partial_{\xi} a\right)\left(x, \xi, x_{1} \varepsilon^{-\alpha}\right)\right) \\
+\varepsilon^{1-2 \alpha} T^{*} \mathrm{op}_{1}\left(\xi_{1} \partial_{y} a\left(x_{1} \varepsilon^{\alpha}, x_{2}, \xi_{1} \varepsilon^{1-\alpha}, \xi_{2} \varepsilon, x_{1}\right)\right) T+o(1) .
\end{array}
\end{gathered}
$$

Here we used that there exists a constant $C$ such that on the support of $a\left(x_{1} \varepsilon^{\alpha}, x_{2}, \xi_{1} \varepsilon^{1-\alpha}, \xi_{2} \varepsilon, x_{1}\right)$, we have $\left|x_{1}\right| \leq C$, therefore $\frac{1}{\varepsilon} \mathrm{e}^{1 /\left(x_{1} \varepsilon^{\alpha}\right)}$ goes to 0 uniformly, as $\varepsilon$ goes to 0 .

This implies that $\left(K_{\varepsilon} \psi^{\varepsilon}(t), \psi^{\varepsilon}(t)\right)$ is uniformly bounded. Therefore, by Ascoli theorem, considering a dense subset of $\mathcal{C}_{0}^{\infty}\left(\mathbb{R}^{2 d+1}, \mathbb{C}^{2,2}\right)$, and then arguing by diagonal extraction, one can find a sequence $\left(\varepsilon_{k}\right)_{k}$ such that, for all $a \in \mathcal{C}_{0}^{\infty}\left(\mathbb{R}^{2 d+1}, \mathbb{C}^{2,2}\right), I_{\varepsilon_{k}, a}(t)$ has a limit as $k$ goes to $+\infty$, uniformly w.r.t. $t$. Besides, observing that

$$
\left(K \psi^{\varepsilon}(t), \psi^{\varepsilon}(t)\right) \underset{\varepsilon \rightarrow 0}{\longrightarrow}\left\langle\xi \cdot \partial_{x} a(x, \xi, y)-\nabla d(x) \cdot \partial_{\xi} a(x, \xi, y), \nu_{t}\right\rangle
$$

by (4.9), we obtain the transport equation (4.8).

\section{A Appendix: Normal form for non degenerated codimen- sion 1 crossing}

We present here a normal form in the spirit of [9], [10] and [15]. We consider a generic class of nondegenerated crossings in two matricial dimensions. We take a $\mathbb{C}^{2,2}$-valued symbol $Q$ and decompose it into a scalar part plus a trace free matrix:

$$
Q(x, \xi)=\phi_{0}(x, \xi) \operatorname{Id}+\left(\begin{array}{cc}
\phi_{1}(x, \xi) & \phi_{2}(x, \xi)+i \phi_{3}(x, \xi) \\
\phi_{2}(x, \xi)-i \phi_{3}(x, \xi) & -\phi_{1}(x, \xi)
\end{array}\right) .
$$

Here the functions $\phi_{0}, \cdots, \phi_{3}$ are real-valued and $\mathcal{C}^{\infty}$. The eigenvalues are the functions

$$
\lambda^{ \pm}(x, \xi)=\phi_{0}(x, \xi) \pm \sqrt{\phi_{1}(x, \xi)^{2}+\phi_{2}(x, \xi)^{2}+\phi_{3}(x, \xi)^{2}} .
$$


The crossing set is the set

$$
\Gamma=\left\{(x, \xi) \in T^{*} \mathbb{R}^{d} ; \phi_{1}(x, \xi)=\phi_{2}(x, \xi)=\phi_{3}(x, \xi)=0\right\} .
$$

We assume that, near some point $\left(x^{*}, \xi^{*}\right) \in \Gamma, \Gamma$ is a codimension one submanifold of $T^{*} \mathbb{R}^{d}$ given by some equation $\gamma(x, \xi)=0$ with $\nabla \gamma \neq 0$ on $\Gamma \cap \Omega_{0}$ where $\Omega_{0}$ is an open neighborhood of $\left(x^{*}, \xi^{*}\right)$. Following [9], [10] and [17], we say that this codimension 1 crossing is non degenerated in $\left(x^{*}, \xi^{*}\right)$ if

$$
\left(\left\{\phi_{0}, \phi_{1}\right\},\left\{\phi_{0}, \phi_{2}\right\},\left\{\phi_{0}, \phi_{3}\right\}\right)\left(x^{*}, \xi^{*}\right) \neq(0,0,0) .
$$

Proposition A.1. If the codimension 1 crossing is non degenerated in $\left(x^{*}, \xi^{*}\right)$, then

$$
\left\{\phi_{0}, \gamma\right\}\left(x^{*}, \xi^{*}\right) \neq 0 .
$$

and the Hamiltonian vector fields $H_{\lambda^{ \pm}}\left(x^{*}, \xi^{*}\right)$ are transverse to the crossing set.

\section{Proof}

The fact that $\gamma=0$ is an equation of the crossing set yields that there exist a neighborhood $\Omega_{1}$ of $\left(x^{*}, \xi^{*}\right)$ and smooth functions $u_{j}(x, \xi), 1 \leq j \leq 3$ such that

$$
\phi_{j}(x, \xi)=\gamma(x, \xi) u_{j}(x, \xi), \quad \forall j \in\{1,2,3\}, \forall(x, \xi) \in \Omega_{1} .
$$

In view of $\gamma\left(x^{*}, \xi^{*}\right)=0$, we obtain

$$
\forall j \in\{1,2,3\},\left\{\phi_{0}, \phi_{j}\right\}\left(x^{*}, \xi^{*}\right)=u_{j}\left(x^{*}, \xi^{*}\right)\left\{\phi_{0}, \gamma\right\}\left(x^{*}, \xi^{*}\right) .
$$

Therefore, the quantities $u_{j}\left(x^{*}, \xi^{*}\right)$ may not be all equal to 0 and $\left\{\phi_{0}, \gamma\right\}\left(x^{*}, \xi^{*}\right) \neq 0$.

Besides, the vector fields $H_{\lambda^{ \pm}}$are transverse to the crossing set if and only if $H_{\lambda^{ \pm}} \gamma\left(x^{*}, \xi^{*}\right) \neq 0$. We observe that

$$
\begin{aligned}
H_{\lambda^{ \pm}} \gamma\left(x^{*}, \xi^{*}\right) & =\left\{\lambda^{ \pm}, \gamma\right\}\left(x^{*}, \xi^{*}\right) \\
& =\left\{\phi_{0}, \gamma\right\}\left(x^{*}, \xi^{*}\right) \pm\left\{\sqrt{\phi_{1}^{2}+\phi_{2}^{2}+\phi_{3}^{2}}, \gamma\right\}\left(x^{*}, \xi^{*}\right) .
\end{aligned}
$$

As $\gamma\left(x^{*}, \xi^{*}\right)=0$ and $\phi_{j}=\gamma u_{j}, 1 \leq j \leq 3$, we get

$$
H_{\lambda^{ \pm}} \gamma\left(x^{*}, \xi^{*}\right)=\left\{\phi_{0}, \gamma\right\}\left(x^{*}, \xi^{*}\right) \neq 0,
$$

whence the proposition.

Before stating the main result, let us recall some basic facts about canonical transforms and Fourier integral operators. The phase space $\mathbb{R}^{2 d}$ has the structure of the cotangent space $T^{*} \mathbb{R}^{d}$ which is a symplectic space endowed with the 2 -form $\omega=\mathrm{d} \xi \wedge \mathrm{d} x$. A canonical transform is a local change of symplectic coordinates, i.e. a local diffeomorphism which preserves the symplectic structure. One can associate with a canonical transform an operator $U$ called Fourier Integral Operator, which is a unitary bounded operator of $L^{2}\left(\mathbb{R}^{d}\right)$ satisfying convenient properties that we explain now. The reader will find in [45] a complete analysis of Fourier Integral Operator, the presentation chosen here is the one of [16].

Once given the canonical transform $\kappa$ in some open set $\Omega$, one constructs a $\mathcal{C}^{1}$ path $\delta \mapsto \kappa(\delta)$, $\delta \in[0,1]$ linking Id to $\kappa$ (see [16, Lemma 1]). The fact that $\kappa(\delta)$ preserves the symplectic structure 
of $T^{*} \mathbb{R}^{d}$ yields that $\frac{\mathrm{d}}{\mathrm{d} \delta} \kappa(\delta) \circ \kappa(\delta)^{-1}$ is a Hamiltonian vector field on $T\left(T \mathbb{R}^{d}\right)$ above $\Omega$. Therefore, there exists a smooth function $f(\delta)$ such that $\kappa(\delta)$ solves

$$
\frac{\mathrm{d}}{\mathrm{d} \delta} \kappa(\delta)=H_{f(\delta)} \kappa(\delta), \quad \kappa(0)=\mathrm{Id}, \quad \kappa(1)=\kappa .
$$

Define the family of unitary operators $U^{\varepsilon}(\delta)$ by

$$
i \varepsilon \frac{\mathrm{d}}{\mathrm{d} \delta} U^{\varepsilon}(\delta)=\mathrm{op}_{\varepsilon}(f(\delta)) U^{\varepsilon}(\delta), \quad U^{\varepsilon}(0)=\mathrm{Id}, \quad \delta \in[0,1] .
$$

Then, we are interested in the operator $U$ defined by

$$
U:=U^{\varepsilon}(1)
$$

This operator satisfies the following formula known as Egorov's Theorem (see Section 2.2 in [16])

$$
\forall a \in \mathcal{C}_{0}^{\infty}\left(\mathbb{R}^{2 d}\right), \quad U^{*} \operatorname{op}_{\varepsilon}(a) U=\mathrm{op}_{\varepsilon}(a \circ \kappa)+O\left(\varepsilon^{2}\right) \text { in } \mathcal{L}\left(L^{2}\left(\mathbb{R}^{d}\right)\right) .
$$

The operator $U$ is a Fourier Integral Operator associated with $\kappa$.

Observe that finding the operator $U$ or finding the function $f$ are equivalent questions; we will use this fact in the proof of the following theorem.

Theorem A.2. If the matrix $Q$ presents a non degenerated codimension 1 crossing in $\left(x^{*}, \xi^{*}\right)$, then for all $N \in \mathbb{N}$, there exist a canonical transform $\kappa_{N}$ from a neighborhood of $\left(x^{*}, \xi^{*}\right)$ into a neighborhood $\Omega$ of 0

$$
\kappa_{N}(x, \xi)=(s, z, \sigma, \zeta), \quad(s, \sigma) \in \mathbb{R}^{2}, \quad(z, \zeta) \in \mathbb{R}^{d-1} \times \mathbb{R}^{d-1}
$$

smooth $\mathbb{C}$-valued functions $\left(\gamma_{j}\right)_{1 \leq j \leq N}$ and smooth matrices $\left(B_{j}(z, \zeta)\right)_{0 \leq j \leq N}$ such that if $U_{N}$ is a Fourier integral operator associated with $\kappa_{N}$ then for all $a \in \mathcal{C}_{0}^{\infty}(\Omega)$, in $\mathcal{L}\left(L^{2}\left(\mathbb{R}^{d}\right)\right)$,

$$
\begin{aligned}
& \mathrm{op}_{\varepsilon}(a)\left(U_{N}^{*} \mathrm{op}_{\varepsilon}\left(B_{N}^{\varepsilon}\right)^{*} \mathrm{op}_{\varepsilon}(Q) \mathrm{op}_{\varepsilon}\left(B_{N}^{\varepsilon}\right) U_{N}\right) \\
&=\mathrm{op}_{\varepsilon}(a) \mathrm{op}_{\varepsilon}\left(\alpha_{0} \sigma \mathrm{Id}+\left(\begin{array}{cc}
s & \varepsilon \gamma_{N}^{\varepsilon}(z, \zeta) \\
\varepsilon \bar{\gamma}_{N}^{\varepsilon}(z, \zeta) & -s
\end{array}\right)\right)+O\left(\varepsilon^{N+1}\right), \\
& B_{N}^{\varepsilon}=B_{0}+\varepsilon B_{1}+\cdots+\varepsilon^{N} B_{N}, \quad \gamma_{N}^{\varepsilon}=\gamma_{1}+\varepsilon \gamma_{2} \cdots+\varepsilon^{N-1} \gamma_{N},
\end{aligned}
$$

where $\alpha_{0}=\operatorname{sgn}\left(\left\{\phi_{0}, \gamma\right\}\left(x^{*}, \xi^{*}\right)\right)$ Besides, $B_{0}^{*} B_{0}=\lambda^{-1} \operatorname{Id}$ with $\lambda\left(x^{*}, \xi^{*}\right) \neq 0$.

Remark A.3. This theorem allows to turn microlocally the equation $\mathrm{op}_{\varepsilon}(Q) \psi^{\varepsilon}=0$ into

$$
\frac{\varepsilon}{i} \partial_{s} u^{\varepsilon}=\left(\begin{array}{cc}
s & \Gamma_{N}^{\varepsilon} \\
\left(\Gamma_{N}^{\varepsilon}\right)^{*} & -s
\end{array}\right) u^{\varepsilon}+O\left(\varepsilon^{N+1}\right)
$$

where $\Gamma_{N}^{\varepsilon}$ is an operator commuting with $s$ and $\partial_{s}$. The solutions of such systems are described in Proposition 7 of 17$]$.

\section{Proof}

We proceed in two steps, following the strategy of 9 : we first work on the classical symbols and find $B_{0}$ and $\kappa$, then we work on the operator level and find $B_{j}$ for $j \geq 1$. Assume that $\left\{\phi_{0}, \gamma\right\}\left(x^{*}, \xi^{*}\right)>0$. 
We first use that $\left\{\phi_{0}, \gamma \sqrt{u_{1}^{2}+u_{2}^{2}+u_{3}^{2}}\right\}\left(x^{*}, \xi^{*}\right)>0$, thus by Lemma 21.3.4 in [28], there exists a positive function $\lambda=\lambda(x, \xi)$ such that

$$
\left\{\lambda \phi_{0}, \lambda \gamma \sqrt{u_{1}^{2}+u_{2}^{2}+u_{3}^{2}}\right\}(x, \xi)=1 .
$$

Then, we set

$$
\sigma(x, \xi)=\lambda(x, \xi) \phi_{0}(x, \xi), \quad s(x, \xi)=\lambda(x, \xi) \gamma(x, \xi) \sqrt{u_{1}^{2}(x, \xi)+u_{2}^{2}(x, \xi)+u_{3}^{2}(x, \xi)} .
$$

We then have

$$
\begin{aligned}
& \lambda(x, \xi) Q(x, \xi) \\
& =\sigma(x, \xi) \operatorname{Id}+\frac{s(x, \xi)}{\sqrt{u_{1}^{2}(x, \xi)+u_{2}^{2}(x, \xi)+u_{3}^{2}(x, \xi)}}\left(\begin{array}{cc}
u_{1}(x, \xi) & u_{2}(x, \xi)+i u_{3}(x, \xi) \\
u_{2}(x, \xi)-i u_{3}(x, \xi) & -u_{1}(x, \xi)
\end{array}\right) .
\end{aligned}
$$

The matrix $\frac{1}{\sqrt{u_{1}^{2}+u_{2}^{2}+u_{3}^{2}}}\left(\begin{array}{cc}u_{1} & u_{2}+i u_{3} \\ u_{2}-i u_{3} & -u_{1}\end{array}\right)$ smoothly diagonalizes in a neighborhood of the point $\left(x^{*}, \xi^{*}\right)$. There exists $B=B(x, \xi)$ smooth and orthogonal such that

$$
\lambda(x, \xi) B^{*}(x, \xi) Q(x, \xi) B(x, \xi)=\sigma(x, \xi) \operatorname{Id}+s(x, \xi)\left(\begin{array}{cc}
1 & 0 \\
0 & -1
\end{array}\right) .
$$

The fact that $\{\sigma, s\}(x, \xi)=1$ yields that by the Darboux theorem, there exists a canonical transform $\kappa_{0}$ such that $\kappa_{0}(x, \xi)=(s, z, \sigma, \zeta)$ and

$$
\left(\lambda B^{*} Q B\right) \circ \kappa_{0}^{-1}(s, z, \sigma, \zeta)=\sigma \operatorname{Id}+s\left(\begin{array}{cc}
1 & 0 \\
0 & -1
\end{array}\right) .
$$

Let us consider now a Fourier integral operator $U_{0}$ associated with $\kappa_{0}$. Setting

$$
B_{0}=\sqrt{\lambda} B
$$

we have

$$
\begin{aligned}
U_{0}^{*} \mathrm{op}_{\varepsilon}\left(B_{0}\right)^{*} \mathrm{op}_{\varepsilon}(Q) \mathrm{op}_{\varepsilon}\left(B_{0}\right) U_{0}=U_{0}^{*} \mathrm{op}_{\varepsilon}\left(B_{0}^{*} Q B_{0}\right) U_{0} & +\varepsilon \mathrm{op}_{\varepsilon}\left(R_{1}\right) \\
& =\mathrm{op}_{\varepsilon}\left(\sigma \operatorname{Id}+s\left(\begin{array}{cc}
1 & 0 \\
0 & -1
\end{array}\right)\right)+\varepsilon \mathrm{op}_{\varepsilon}\left(R_{1}\right)
\end{aligned}
$$

where we have used symbolic calculus and the definition of $U_{0}$. It remains now to get rid of the rest term $R_{1}$ (which is self-adjoint) by modifying the canonical transform $\kappa_{0}$ and the matrix $B_{0}$. We proceed by induction: we suppose that we have find $\kappa_{N}, U_{N}, B_{0}, \cdots, B_{N}$ and $\gamma_{1}, \cdots, \gamma_{N}$ such that microlocally near $(0,0)$ we have in $\mathcal{L}\left(L^{2}\left(\mathbb{R}^{d}\right)\right)$

$$
U_{N}^{*} \mathrm{op}_{\varepsilon}\left(B_{N}^{\varepsilon}\right)^{*} \mathrm{op}_{\varepsilon}(Q) \mathrm{op}_{\varepsilon}\left(B_{N}^{\varepsilon}\right) U_{N}=\mathrm{op}_{\varepsilon}\left(Q_{0}+\Gamma_{N}^{\varepsilon}\right)+\varepsilon^{N+1} \mathrm{op}_{\varepsilon}\left(R_{N}\right)+O\left(\varepsilon^{N+2}\right)
$$

where

$$
Q_{0}(s, \sigma)=\sigma \mathrm{Id}+\left(\begin{array}{cc}
s & 0 \\
0 & -s
\end{array}\right), \quad \Gamma_{N}^{\varepsilon}=\left(\begin{array}{cc}
0 & \gamma_{N}^{\varepsilon} \\
\bar{\gamma}_{N}^{\varepsilon} & 0
\end{array}\right)
$$


We decompose $R_{N}$ as

$$
R_{N}=p_{N} \operatorname{Id}+\left(\begin{array}{cc}
d_{N} & v_{N} \\
\bar{v}_{N} & -d_{N}
\end{array}\right)=\tilde{R}_{N}+\left(\begin{array}{cc}
0 & \gamma_{N+1} \\
\bar{\gamma}_{N+1} & 0
\end{array}\right)
$$

where $\gamma_{N+1}(z, \zeta)=v_{N}(0, z, 0, \zeta)$, so that for some smooth functions $g$ and $h$,

$$
v_{N}(s, z, \sigma, \zeta)=\gamma_{N+1}(z, \zeta)+s g(s, z, \sigma, \zeta)+\sigma h(s, z, \sigma, \zeta) .
$$

We claim that we can find a function $f$ and a matrix $D$ such that

$$
\left\{f, Q_{0}\right\}+D^{*} Q_{0}+Q_{0} D+\tilde{R}_{N}=0 .
$$

Let us postpone the proof of this claim at the end of the proof.

We then choose

$$
B_{N+1}=B_{0} D
$$

and we compose $\kappa_{N}$ with a perturbation of the identity constructed from $f$. We use a family of canonical transforms $\chi^{\varepsilon}(\delta)$ for $\delta \in[0,1]$ which satisfy

$$
\frac{\mathrm{d}}{\mathrm{d} \delta} \chi^{\varepsilon}(\delta)=H_{\varepsilon^{N+1} f} \circ \chi^{\varepsilon}(\delta), \quad \chi^{\varepsilon}(0)=\mathrm{Id} .
$$

If $U^{\varepsilon}(\delta)$ is a Fourier integral operator associated with $\chi^{\varepsilon}(\delta)$, we have by (A.2)

$$
\frac{\mathrm{d}}{\mathrm{d} \delta}\left(U^{\varepsilon}(\delta)^{*} \operatorname{op}_{\varepsilon}(a) U^{\varepsilon}(\delta)\right)=\varepsilon^{N+1} U^{\varepsilon}(\delta)^{*} \operatorname{op}_{\varepsilon}(\{f, a\}) U^{\varepsilon}(\delta)+O\left(\varepsilon^{N+2}\right) \text { in } \mathcal{L}\left(L^{2}\left(\mathbb{R}^{d}\right)\right) .
$$

Consider

$$
\begin{array}{r}
C^{\varepsilon}(\delta)=U^{\varepsilon}(\delta)^{*}\left[U_{N}^{*} \mathrm{op}_{\varepsilon}\left(\left(B_{N}^{\varepsilon}\right)^{*}+\delta \varepsilon^{N+1} B_{N+1}^{*}\right) \mathrm{op}_{\varepsilon}(Q) \mathrm{op}_{\varepsilon}\left(B_{N}^{\varepsilon}+\delta \varepsilon^{N+1} B_{N+1}\right) U_{N}\right. \\
\left.-(1-\delta) \varepsilon^{N+1} \mathrm{op}_{\varepsilon}\left(\tilde{R}_{N}\right)\right] U^{\varepsilon}(\delta) .
\end{array}
$$

Setting

$$
U_{N+1}=U_{N} \circ U^{\varepsilon}(1)
$$

we have, microlocally near $(0,0)$

$$
\begin{gathered}
C^{\varepsilon}(0)=\operatorname{op}_{\varepsilon}\left(Q_{0}+\Gamma_{N+1}^{\varepsilon}\right)+O\left(\varepsilon^{N+2}\right) \\
\left.C^{\varepsilon}(1)=U_{N+1}^{*} \operatorname{op}_{\varepsilon}\left(\left(B_{N+1}^{\varepsilon}\right)^{*}\right) \operatorname{op}_{\varepsilon}(Q) \operatorname{op}_{\varepsilon}\left(B_{N+1}^{\varepsilon}\right)\right) U_{N+1} .
\end{gathered}
$$

Besides, by symbolic calculus, we obtain in $\mathcal{L}\left(L^{2}\left(\mathbb{R}^{d}\right)\right)$

$$
\begin{aligned}
C^{\varepsilon}(\delta)=U^{\varepsilon}(\delta)^{*} & {\left[\operatorname{op}_{\varepsilon}\left(Q_{0}+\Gamma_{N}^{\varepsilon}+\delta \varepsilon^{N+1}\left(B_{N+1}^{*} Q B_{0}+B_{0}^{*} Q B_{N+1}\right)+\delta \varepsilon^{N+1} \tilde{R}_{N}\right)\right] U^{\varepsilon}(\delta) } \\
+O\left(\varepsilon^{N+2}\right) & +U^{\varepsilon}(\delta)^{*}\left[\operatorname{op}_{\varepsilon}\left(Q_{0}+\Gamma_{N}^{\varepsilon}+\varepsilon^{N+1} \delta\left(D^{*} Q_{0}+Q_{0} D\right)+\delta \varepsilon^{N+1} \tilde{R}_{N}\right)\right] U^{\varepsilon}(\delta)+O\left(\varepsilon^{N+2}\right)
\end{aligned}
$$

where we have used $B_{0}^{*} Q B_{0}=Q_{0}$. Therefore, in view of $(\mathrm{A} .2$,

$$
\frac{\mathrm{d}}{\mathrm{d} \delta} C^{\varepsilon}(\delta)=\varepsilon^{N+1} U^{\varepsilon}(\delta)^{*} \operatorname{op}_{\varepsilon}\left(\left\{f, Q_{0}\right\}+D^{*} Q_{0}+Q_{0} D+\tilde{R}_{N}\right) U^{\varepsilon}(\delta)+O\left(\varepsilon^{N+2}\right)=O\left(\varepsilon^{N+2}\right)
$$


in $\mathcal{L}\left(L^{2}\left(\mathbb{R}^{d}\right)\right)$. Integrating between $\delta=0$ and $\delta=1$, we get

$$
C^{\varepsilon}(1)=C^{\varepsilon}(0)+O\left(\varepsilon^{N+2}\right) \text { in } \mathcal{L}\left(L^{2}\left(\mathbb{R}^{d}\right),\right.
$$

which gives the next step of the induction argument.

It remains to prove that one can solve (A.4). We write

$$
\tilde{R}_{N}=p_{N}(0, z, 0, \zeta) \mathrm{Id}+d_{N}(0, z, 0, \zeta) J+s R_{N}^{(1)}+\sigma R_{N}^{(2)}, \quad J=\left(\begin{array}{cc}
1 & 0 \\
0 & -1
\end{array}\right) .
$$

In view of $\left\{f, Q_{0}\right\}=-\partial_{s} f \operatorname{Id}+\partial_{\sigma} f J$, setting

$$
f(s, z, \sigma, \zeta)=\tilde{f}(s, z, \sigma, \zeta)+s p_{N}(0, z, 0, \zeta)-\sigma d_{N}(0, z, 0, \zeta)
$$

we are reduced to find $\tilde{f}$ and $D$ such that

$$
\left\{\tilde{f}, Q_{0}\right\}+D^{*} Q_{0}+Q_{0} D+s R_{N}^{(1)}+\sigma R_{N}^{(2)}=0 .
$$

For this, we consider $Q_{\delta}=Q_{0}+\delta\left(s R_{N}^{(1)}+\sigma R_{N}^{(2)}\right)$ for small $\delta$. The strategy is to prove that there exist a matrix $B_{\delta}$ and a canonical transform $\kappa_{\delta}$ such that

$$
B_{\delta}^{*}\left(Q_{\delta} \circ \kappa_{\delta}\right) B_{\delta}=Q_{0} .
$$

Differentiating this last relation with respect to $\delta$ and putting $\delta=0$, one solves the homological equation A.5. Let us consider the symbol $Q_{\delta}$. We have

$$
Q_{\delta}=\sigma\left(\mathrm{Id}+\delta R_{N}^{(2)}\right)+s\left(J+\delta R_{N}^{(1)}\right) .
$$

The matrix $\mathrm{Id}+\delta R_{N}^{(2)}$ is symmetric and invertible for $\delta$ small enough in a neighborhood of 0 . Thus, if $C_{1}=\left(\sqrt{\mathrm{Id}+\delta R_{N}^{(2)}}\right)^{-1}$ then $C_{1}=C_{1}^{*}$ and

$$
C_{1} Q_{\delta} C_{1}=\sigma \mathrm{Id}+s C_{1}\left(J+\delta R_{N}^{(1)}\right) C_{1}
$$

We observe that

$$
C_{1}\left(J+\delta R_{N}^{(1)}\right) C_{1}=J+O(\delta), \operatorname{tr}\left(C_{1}\left(J+\delta R_{N}^{(1)}\right) C_{1}\right)=O(\delta),
$$

therefore, we can write $C_{1}\left(J+\delta R_{N}^{(1)}\right) C_{1}=J+\delta R_{N}^{(3)}$ and

$$
C_{1} Q_{\delta} C_{1}=\left(\sigma+s \delta \operatorname{tr}\left(R_{N}^{(3)}\right)\right) \mathrm{Id}+s\left(J+\delta R_{N}^{(4)}\right), \operatorname{tr}\left(R_{N}^{(4)}\right)=0 .
$$

One easily see that the matrix $C_{1} Q_{\delta} C_{1}$ has a codimension 1 crossing as long as $\delta$ is small enough so that the eigenvalues of $\delta R_{N}^{(4)}$ are smaller than 1 . Therefore, by the first step of our proof, there exist a matrix $B_{1}$ and a canonical transform $\kappa(\delta)$ such that

$$
\left(B_{1}^{*} C_{1} Q_{\delta} C_{1} B_{1}\right) \circ \kappa(\delta)=Q_{0} .
$$

Setting $B(\delta)=\left(C_{1} B_{1}\right) \circ \kappa(\delta)$, one closes the proof of the normal form result. 


\section{References}

[1] P. Braam, H. Duistermaat: Normal forms of real symmetric systems with multiplicity. Indag. Math.; N.S. 4 (1993), no. 4, p. 407-421.

[2] M. Brassart: Limite semi-classique de transformées de Wigner dans des milieux périodiques ou aléatoires. Thèse de l'Université de Nice Sophia Antipolis (2002).

[3] N. Burq: Semiclassical estimates for the resolvent in non trapping geometry. Int. Math. Res. Notices 5 (2002), p. 221-241.

[4] N. Burq: Smoothing effect for Schrödinger boundary value problem. Duke Mathematical Journal, 123 (2004), no. 2, p. 221-241.

[5] N. Burq, G. Lebeau: Mesures de défaut de compacité, application au système de Lamé. Ann. Sci. École Norm. Sup. (4) 34 (2001), no. 6, p. 817-870.

[6] R. Carles, C. Fermanian Kammerer, N. Mauser, H.-P. Stimming: On the time evolution of Wigner measures for Schrödinger equations (to appear in CPAA).

[7] F. Castella, A. Knauf, Th. Jecko: Semiclassical resolvent estimates for Schrödinger operators with Coulomb singularities. Annales Henri Poincaré, 9 (2008), p. 775-815. .

[8] F. Castella, Th. Jecko: Besov estimates in the high-frequency Helmholtz equation, for a nontrapping and $C^{2}$ potential. J. Diff. Eq., 228 (2006), no. 2, 440-485.

[9] Y. Colin de Verdière: The level crossing problem in semi-classical analysis. I. The symmetric case. Proceedings of the International Conference in Honor of Frédéric Pham (Nice, 2002). Ann. Inst. Fourier (Grenoble) $\mathbf{5 3}$ (2003), no. 4, p. 1023-1054.

[10] Y. Colin de Verdière: The level crossing problem in semi-classical analysis. II. The Hermitian case. Ann. Inst. Fourier (Grenoble) $\mathbf{5 4}$ (2004), no. 5, p. 1423-1441.

[11] H. L. Cycon, R. Froese, W. Kirsch, B. Simon: Schrödinger operators with application to quantum mechanics and global geometry. Springer-Verlag (1987).

[12] M. Dimassi, J. Sjöstrand: Spectral Asymptotics in the Semi-Classical Limit. London Math. Soc. Lecture Note Series 268, Cambridge University Press (1999).

[13] T. Duyckaerts: Inégalités de résolvante pour l'opérateur de Schrödinger avec potentiel multipolaire critique. (French.) [Resolvent estimates for the Schrödinger operator with critical multipolar potential] Bull. Soc. Math. France 134 (2006), no. 2, p. 201-239.

[14] C. Fermanian Kammerer: Semiclassical analysis of generic codimension 3 crossings. Int. Math. Res. Not. 45 (2004), p. 2391-2435.

[15] C. Fermanian Kammerer: Wigner measures and molecular propagation through generic energy level crossings, Rev. Math. Phys. 15 (2003), no. 10 , p. 1285-1317.

[16] C. Fermanian Kammerer, P. Gérard: Mesures semi-classiques et croisements de modes. Bull. Soc. math. France 130 (2002), no 1, p. 123-168.

[17] C. Fermanian Kammerer, P. Gérard: A Landau-Zener formula for non-degenerated involutive codimension 3 crossings. Ann. Henri Poincaré 4 (2003), no 3, p. 514-552. 
[18] C. Fermanian Kammerer, V. Rousse: Resolvent estimates for a Schrödinger operator with matrix-valued potential presenting eigenvalue crossings. Application to Strichartz estimates, Comm. in Part. Diff. Eq. 33 (2008), no. 1, p. 19-44.

[19] R. Froese, I. Herbst: Exponential bounds and absence of positive eigenvalues for $N$-body Schrödinger operators. Comm. Math. Phys. 87, 3 (1982/1983), p. 429-447.

[20] S. Fujiié, C. Lasser, L. Nédélec : Semiclassical resonances for a two-level Schrödinger operator with a conical intersection. Preprint.

[21] C. Gérard: Semiclassical resolvent estimates for two and three-body Schrödinger operators. Comm. Partial Differential Equations 15 (1990), no. 8, p. 1161-1178.

[22] C. Gérard, A. Martinez: Principe d'absorption limite pour des opérateurs de Schrödinger à longue portée. (French) [The limiting absorption principle for long-range Schrödinger operators] C. R. Acad. Sci. Paris Sér. I Math. 306 (1988), no. 3, p. 121-123.

[23] P. Gérard: Mesures semi-classiques et ondes de Bloch, Séminaire sur les Équations aux Dérivées Partielles, 1990-1991, Exp. No. XVI, 19 (1991).

[24] P. Gérard, E. Leichtnam: Ergodic properties of eigenfunctions for the Dirichlet problem, Duke Math. J. 71, 2 (1993), p. 559-607.

[25] P. Gérard, P. Markowich, N. Mauser, and F. Poupaud: Homogenization limits and Wigner transforms. Commun. Pure Appl. Math. 50, 4 (1997), p. 323-379.

[26] P. Gérard, P. Markowich, N. Mauser, and F. Poupaud: Erratum: "Homogenization limits and Wigner transforms Commun. Pure Appl. Math. 50, 4 (1997), p. 323-379." Commun. Pure Appl. Math. 53 (2000), no. 2 p. 280-281.

[27] G. A. Hagedorn: Molecular propagation through electron energy level crossings. Memoirs AMS, 536, no. 111 (1994).

[28] L. Hörmander: The analysis of linear Partial Differential Operators III. Springer-Verlag, 1985.

[29] T. Jecko: Estimations de la résolvante pour une molécule diatomique dans l'approximation de Born-Oppenheimer. Comm. Math. Phys. 195 (1998) no. 3, p. 585-612.

[30] T. Jecko: Semiclassical resolvent estimates for Schrödinger matrix operators with eigenvalues crossings. Math. Nachr., 257 (2003), no. 1, p. 36-54.

[31] T. Jecko: From classical to semiclassical non-trapping behaviour. C. R. Acad. Sci. Paris, Ser. I 338 (2004), p. 545-548.

[32] T. Jecko: Non-trapping condition for semiclassical Schrödinger operators with matrix-valued potentials. Math. Phys. Electronic Journal 11 (2005), no. 2.

Erratum: Math. Phys. Electronic Journal, No. 3, vol. 13, 2007.

[33] U. Karlsson: Semi-Classical approximations of Quantum Mechanical Problems. Doctoral Dissertation (2002), Royal Institute of Technology, Stockolm.

[34] J-L. Krivine: Introduction to axiomatic set theory. Translated from the French by David Miller D. Reidel Publishing Co., Dordrecht; Humanities Press, New York 1971, 98 pp. 
[35] J-L. Krivine: Théorie des ensembles. Cassini, Paris 2007.

[36] G. Lebeau: Equation des ondes amorties, in Algebraic and geometric methods in mathematical physics (Kaciveli, 1993), Math. Phys. Stud., vol. 19, Kluwer Acad. Publ., Dordrecht, 1996, p. $73-109$.

[37] P.-L. Lions, T. Paul: Sur les mesures de Wigner, Revista Matemática Iberoamericana 9 (1993), no. 3 , p. 553-618.

[38] A. Martinez: An introduction to semiclassical and microlocal analysis. Universitext Springer, 2002.

[39] R. B. Melrose, J. Sjöstrand: Singularities of boundary value problems. I. Comm. Pure Appl. Math. 31 (1978), no. 5, p. 593-617.

[40] L. Miller: Propagation d'onde semi-classiques à travers une interface et mesures 2-microlocales. Thèse de l'Ecole Polytechnique (1996).

[41] E. Mourre: Absence of singular continuous spectrum for certain self-adjoint operators. Comm. Math. Phys. 78 (1981), p. 391-408.

[42] L. Nédélec: Resonances for matrix Schrödinger operators. Duke Math. J. 106 (2001), no. 2, p. 209-236.

[43] M. Reed, B. Simon: Method of Modern Mathematical Physics, Tome II : Fourier Analysis, Self-adjointness. Academic Press 1979.

[44] M. Reed, B. Simon: Method of Modern Mathematical Physics, Tome IV : Analysis of operators. Academic Press 1979

[45] D. Robert: Autour de l'approximation semi-classique. Birkhaüser, 1983.

[46] D. Robert, H. Tamura: Semiclassical estimates for resolvents and asymptotics for total crosssection. Ann. IHP 46 (1987), p. 415-442.

[47] A. Vasy, M. Zworski: Semiclassical estimates in asymptotically Euclidean scattering. Comm. Math. Phys. 212 (2000), no. 1, p. 205-217.

[48] R. L. Vaught: Set Theory. An introduction. Birkhäuser Boston, 1995.

[49] X.P. Wang: Time-decay of scattering solutions and classical trajectories. Ann. Inst. H. Poincaré Phys. Théor. 47 (1987), no. 1, p. 25-37.

[50] X.P. Wang: Semiclassical resolvent estimates for $N$-body Schrödinger operators. J. Funct. Anal. 97 (1991), p. 466-483.

Thomas Duyckaerts, Université de Cergy Pontoise, 2 av. A. Chauvin, BP 222, 95302 CergyPontoise Cedex, France.

Thomas. Duyckaerts@u-cergy.fr

Clotilde Fermanian Kammerer, Université Paris Est, UFR des Sciences et Technologie, 61, avenue du Général de Gaulle, 94010 Créteil Cedex, France.

Clotilde.Fermanian@univ-paris12.fr

Thierry Jecko, Université de Cergy Pontoise, 2 av. A. Chauvin, BP 222, 95302 Cergy-Pontoise Cedex, France.

Thierry.Jecko@u-cergy.fr 